\title{
The Multiobjective Constraint Fault-Tolerant Control of Event-Triggered Nonuniform Transmission for Networked T-S Fuzzy System
}

\author{
Ya-jie $L^{\mathbf{i}^{1,2}}$ and Wei $\mathrm{Li}^{1}$ \\ ${ }^{1}$ College of Electrical and Information Engineering, Lanzhou University of Technology, Lanzhou 730050, China \\ ${ }^{2}$ Automation Institute of Lanzhou Petrochemical of PetroChina, Lanzhou 730060, China \\ Correspondence should be addressed to Wei Li; liwei@lut.cn
}

Received 13 April 2016; Revised 19 September 2016; Accepted 5 October 2016

Academic Editor: Mohammad D. Aliyu

Copyright (C) 2016 Y.-j. Li and W. Li. This is an open access article distributed under the Creative Commons Attribution License, which permits unrestricted use, distribution, and reproduction in any medium, provided the original work is properly cited.

Considering the actuator saturation, the problem of multiobjective constraint fault-tolerant control is studied for networked Takagi-Sugeno (T-S) fuzzy system with nonuniform transmission period under the discrete event-triggered communication scheme (DETCS). A closed-loop T-S fuzzy model, which includes numerous factors such as actuator saturation, actuator failure, event-triggered condition, nonuniform transmission period, and time-delay, is established by transferring the effect caused by the nonuniform update period on the system performance into the effect on the system time-delay. Two stability criterions and two codesign methods for the networked closed-loop failure T-S fuzzy system are developed based on a discontinuous Lyapunov-Krasovskii function, a kind of reciprocally convex technology, a kind of linear convex combination, Jessen inequality, and Wirtinger's inequality. A feasible experimental solution is presented to verify the effectiveness of the proposed method.

\section{Introduction}

The T-S fuzzy model is known as an effective way of expressing nonlinear system, in which the model expresses the nonlinear system into a combination of several linear subsystems with different membership degrees, applying numerous mature methods of the linear system into the nonlinear system $[1,2]$. Therefore, a large class of nonlinear systems can be well approximated by T-S fuzzy models because these models can easily present the dynamic performance of the complex system [3], like wastewater treatment plant [3], a nonlinear model of the vehicle [4], and so on [5]. There are numerous results [6-9] of analysis and synthesis for the nonlinear networked control system (NCS) described by T-S fuzzy model because of the successful application of the network in different industries. Furthermore, these results not only possess important theoretical significance but also have special practical value.
Based on the existing nonlinear NCS research results on T-S fuzzy model, most of the work was studied by adopting a periodic time-triggered communication scheme (PTTCS). Although the system design under PTTCS is easy to analyze and implement, the scheme could lead to a few problems such as network resource waste and network congestion. Essentially, the NCS is an integrated body of control and communication, whereas the PTTCS considers the control and communication in a fragmented way in the process of analysis and synthesis. Thus, the PTTCS cannot simultaneously consider the system control quality and the network service quality for system design. The appearance of DETCS offers an effective way to solve the preceding problems. Saving network communication resources will be obvious because only the data which satisfies certain eventtriggered conditions will be transmitted by the network. Furthermore, the DETCS unifies the control attribute and the communication attribute into one framework, which is the basis for the implementation of the codesign between 
control and communication. Recently, the nonlinear NCS control problems under DETCS are gradually attracting more attention by utilizing the T-S fuzzy model [10-12]. Reference [11] presents a kind of DETCS for a class of networked T-S fuzzy systems. In the existing study results under DETCS, a nonuniform transmission period exists when the event trigger selects the transmission data. These problems are usually studied by using the continuous method in one period. The nonuniform transmission period generated by the event trigger is essentially an integer multiple of the sample period; thus, the nonuniform transmission control problem under DETCS is regarded as the nonuniform sample control problem. Regardless of whether there is an equal sample period in the sampler or not, we can bind the event generator with the sampler and consider both the event generator and the sampler as a virtual nonuniform sampler. Therefore, NCS is essentially a typical sample-data hybrid system which can possibly analyze and synthesize the system by adopting the related study method of the sample-data control system.

By studying the method for the sample-data control system, all the methods can be generally summarized into three categories [13]. The first method is converting the continuous time plant into discrete one and designing the discrete controller under the discrete system control theory. In the second method, the continuous time controller is designed based on the continuous system control theory and then converting the continuous time controller into discrete one [14]. The effect generated by the nonuniform update period on the system performance is transferred into the effect of the system time-delay during the third method, and the related problems are studied using excellent and mature methods in the time-delay system $[13,15]$. The conclusion is drawn by further analyzing the three preceding methods. Obtaining the nonlinear discrete system model by using the first method is difficult. Selecting a smaller sample period to implement better approximation for continuous time controller in the second method is necessary. The two preceding methods should either consider the discrete sample period before solving the controller or consider the discrete sample period after obtaining the controller. Moreover, the third method is carried out based on the relative mature time-delay system theory, which neither needs to convert the continuous control plant into discrete one nor needs to convert the continuous time controller into discrete one and only needs to analyze the effect of the nonuniform sample period on the system performance by the dealing method of the input delay. Therefore, the nonuniform sample system control problem based on the third method has gradually attracted more attention $[13,15,16]$.

The stability and the performances of the system cannot be ensured with the general control law if any component of the system has a fault $[3,4]$. A kind of control technology, which is known as the fault-tolerant control, has been proposed for this situation to guarantee the stability and acceptable performances. In addition, because of the complexness and uncertainties of NCS, security has become the focus of attention. In the last ten years, the fault-tolerant control technology played an important role in improving the safety and reliability in NCS, thereby attracting numerous scholars [17-19] who devoted numerous efforts to the research of faulttolerant control in NCS. Compared with the fault-tolerant control technology in a traditional control system, the faulttolerant technology of NCS can solve a few problems caused by network communication such as data dropout, time-delay, and disorder [20, 21]. Apart from the general fault-tolerant control in NCS, the multiobjective constraint fault-tolerant control of NCS has also become the focus of academic research [22-24]. Aside from stability, the failure system should possess a few other control performances such as $\alpha$ safety degree [25], $H_{\infty}$-performance, $H_{2}$-performance, and pole assignment.

Thus, few circumstances of the nonuniform transmission sample-data hybrid system under the DETCS were studied by using the methods of nonuniform sample-data control system for the nonlinear NCS based on the T-S fuzzy model. Afterwards, the case simulated the interest of studying the codesign between nonuniform transmission multiobjective constraint fault-tolerant control for nonlinear NCS and network communication.

The study has three main contributions:

(1) A closed-loop T-S fuzzy model is established. The model includes actuator saturation, actuator failure, event-triggered condition, nonuniform transmission period, and time-delay. In building the model, the effect of nonuniform transmission period on transmission time-delay is thoroughly analyzed in the present study by utilizing the research method in the nonuniform sample-data system.

(2) Two multiobjective constraint sufficient conditions and two multiobjective codesign methods are derived for the T-S fuzzy NCS. The multiobjective constraint includes $\alpha$-safety degree, $H_{\infty}$-performance index, $H_{2}$-performance index, and as little as possible network communication resource occupancy. The proposed method provides a better dynamic performance for the controlled plant while simultaneously occupying fewer network communications for the entire system.

(3) The codesign method processes less conservatism during solving the controller and event-triggered weight matrix. The discontinuous Lyapunov-Krasovskii function which can allow the system in tolerating a bigger time-delay under multiobjective constraint is adopted. The bigger time-delay makes the codesign easier. The relevant content is analyzed in the simulation experiment.

Notations. $R^{n}$ represents the $n$-dimensional real vector space; $R^{m \times n}$ is the set of all $(m \times n)$-dimensional real matrices; $A>$ $0(\geq 0)$ indicates that the matrix is a positive (nonnegative) definite; $\operatorname{diag}\{\cdots\}$ refers to the block-diagonal matrix; $I$ is the identity matrix of the appropriate dimension; and $A^{T}$ is the transpose of the matrix $A$. In symmetric block matrices, “*” is used as an ellipsis for terms induced by symmetry; matrices, if not explicitly stated, are assumed to possess appropriate dimensions. 


\section{The Statement of the Problem and Preparation}

2.1. The Description of the Plant. The typical class of nonlinear controlled plant can be expressed as

$$
\begin{aligned}
\dot{x}(t) & =F(x(t), \text { sat }(u(t)), w(t)) \\
& =f(x(t))+g(x(t))(\text { sat }(u(t)), w(t)), \\
z_{1}(t) & =C_{1}(x(t)), \\
z_{2}(t) & =C_{2}(x(t)),
\end{aligned}
$$

where $x(t) \in R^{n}$ and $u(t) \in R^{m}$ denote the state vector and the control input, respectively. The function sat(.) : $R^{m} \rightarrow R^{m}$ denotes the standard multivariable saturation function defined as $\operatorname{sat}(u)=\left[\operatorname{sat}\left(u_{1}\right), \operatorname{sat}\left(u_{2}\right), \ldots, \operatorname{sat}\left(u_{m}\right)\right]^{T}$, and $\operatorname{sat}\left(u_{i}\right)=\operatorname{sign}\left(u_{i}\right) \min \left\{1,\left|u_{i}\right|\right\} . z_{1}(t)$ and $z_{2}(t)$ are two regulated outputs. $w(t) \in L_{2}[0, \infty)$ is the external disturbance. $f(x(t)), g(x(t)), C_{1}(x(t))$, and $C_{2}(x(t))$ are the unknown nonlinear functions based on $x(t)$.

T-S fuzzy model can be regarded as a universal approximator for the general nonlinear system. Considering the following nonlinear systems represented by several simple local linear dynamic systems with their linguistic description, the controlled plant with the actuator saturation constraint can be described based on the following if-then rule.

$$
\begin{aligned}
& \text { If } \theta_{1}(t) \text { is } M_{i 1} \ldots \text { and } \theta_{g}(t) \text { is } M_{i g}, \text { then } \\
& \begin{aligned}
\dot{x}(t)= & \left(A_{i}+\Delta A_{i}\right) x(t)+\left(B_{i}+\Delta B_{i}\right) \text { sat }(u(t)) \\
& +B_{w i} w(t), \\
z_{1}(t)= & C_{i 1} x(t), \\
z_{2}(t)= & C_{i 2} x(t),
\end{aligned}
\end{aligned}
$$

where $\theta(t)=\left[\theta_{1}(t), \theta_{2}(t), \ldots, \theta_{g}(t)\right]^{T}$ denotes the premise variables which assume that $\theta(t)$ is either a given function or a function of $x(t)$ which does not depend on $u(t) ; M_{i j}(i=$ $1,2, \ldots, r ; j=1,2, \ldots, r)$ is the fuzzy set, and $r$ is the number of if-then rules; $A_{i} \in R^{n \times n}$ and $B_{i} \in R^{n \times m}$ are the system matrix and the input matrix, respectively. Moreover, $\Delta A_{i}, \Delta B_{i}(i=1,2, \ldots, r)$, which are assumed as normbounded, denote the uncertainty matrix of system parameters. $\Delta A_{i}, \Delta B_{i}(i=1,2, \ldots, r)$ are time-varying and are satisfying

$$
\left[\Delta A_{i}, \Delta B_{i}\right]=\operatorname{MF}(t)\left[E_{a i}, E_{b i}\right]
$$

where $M, E_{a i}$, and $E_{b i}$ are the real constant matrices with appropriate dimensions; $F(t)$ is an unknown time-varying continuous matrix function with real values and the elements of $F(t)$ are Lebesgue measurable. $F(t)$ satisfies $F^{T}(t) F(t) \leq I$.
The fuzzy system is the weighted average of every output of the subsystem; namely,

$$
\begin{array}{r}
\dot{x}(t)=\sum_{i=1}^{r} h_{i}(\theta(t))\left[\left(A_{i}+\Delta A_{i}\right) x(t)\right. \\
\left.+\left(B_{i}+\Delta B_{i}\right) \text { sat }(u(t))+B_{w i} w(t)\right] \\
z_{1}(t)=\sum_{i=1}^{r} h_{i}(\theta(t)) C_{i 1} x(t), \\
z_{2}(t)=\sum_{i=1}^{r} h_{i}(\theta(t)) C_{i 2} x(t)
\end{array}
$$

where

$$
\begin{aligned}
h_{i}(\theta(t)) & =\frac{w_{i}(\theta(t))}{\sum_{i=1}^{r} w_{i}(\theta(t))} \geq 0, \\
\sum_{i=1}^{r} w_{i}(\theta(t))>0, & \forall t \geq 0, \\
\sum_{i=1}^{r} h_{i}(\theta(t)) & =1, \quad(i=1,2, \ldots, r), \\
w_{i}(\theta(t)) & =\prod_{s=1}^{g} M_{i s}\left(\theta_{s}(t)\right),
\end{aligned}
$$

and $M_{i s}\left(\theta_{s}(t)\right)$ is the degree of the membership for the variable $\theta_{s}(t)$ which belongs to the fuzzy set $M_{i s}$.

2.2. The Analysis of the Effective Nonuniform Transmission Sequence for the Sample Signal under DETCS. This paper adopted DETCS for studying nonuniform transmission multiobjective fault-tolerant control problem of nonlinear NCS with actuator saturation to reduce the network resource waste and implement the codesign between control and communication. The structure chart is expressed as in Figure 1.

Figure 1 shows that there are the controlled plants based on T-S fuzzy model, sensor, sampler, event generator, fuzzy fault-tolerant controller, zero-order holder, and actuator. The sampler is driven by the clock whereas the controller and the zero-order holder are both driven by the event. In contrast to traditional NCS, the sampled data passes the event generator before transmission by the network. The function of the event generator is to determine whether the latest sample signal should be transmitted to the controller. The discrete eventtriggered condition is presented as follows:

$$
\begin{aligned}
& {\left[x\left(i_{k} \hbar\right)-x\left(t_{k} \hbar\right)\right]^{T} \Phi\left[x\left(i_{k} \hbar\right)-x\left(t_{k} \hbar\right)\right]} \\
& \quad \leq \sigma x^{T}\left(t_{k} \hbar\right) \Phi x\left(t_{k} \hbar\right),
\end{aligned}
$$

where $\Phi$ is a symmetric positive definite matrix which is the weight matrices of the DETCS to be designed; $\sigma>0$ is the event-triggered parameter; $x\left(i_{k} \hbar\right)$ and $x\left(t_{k} \hbar\right)$ denote 


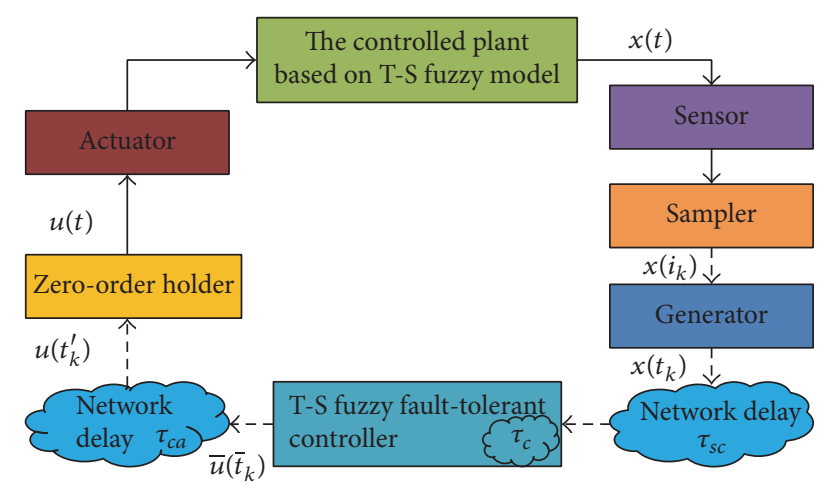

$\longrightarrow$ Continuous signal

$-\rightarrow$ Discrete signal

FIGURE 1: The control structure chart of nonuniform transmission nonlinear NCS under DETCS.

the current sampled data and the latest transmission data, respectively.

Suppose that $\left\{i_{k} \hbar\right\}(k=0,1,2, \ldots)$ denotes the sample instant sequence in the rear end of the sampler and $\hbar$ is the sample period. $\left\{t_{k} \hbar\right\}(k=0,1,2, \ldots)$ denotes the data transmission instant sequence in the rear end of the generator. Simultaneously, suppose that the data to be transmitted in the nonlinear NCS will arrive at the end of the holder after a piece of comprehensive time-delay $\tau_{t_{k} \hbar}$. We set the comprehensive time-delay as $\tau_{t_{k} \hbar}=\tau_{s c}^{t_{k} \hbar}+\tau_{c a}^{t_{k} \hbar}+\tau_{c}^{t_{k} \hbar}$ at the instant $t_{k} \hbar$, where $\tau_{s c}^{t_{k} \hbar}$ and $\tau_{c a}^{t_{k} \hbar}$ denote the transmission time-delay from the generator to the controller and from the controller to the actuator front end, respectively, and $\tau_{c}^{t_{k} \hbar}$ denote the calculation time-delay. The data transmission instant sequence at the end of the controller is $\left\{\bar{t}_{k} \hbar=t_{k} \hbar+\right.$ $\left.\tau_{s c}^{t_{k} \hbar}+\tau_{c}^{t_{k} \hbar}\right\}(k=0,1,2, \ldots)$; the updating instant sequence at the front end of the holder is $\left\{\underline{t}_{k} \hbar=t_{k} \hbar+\tau_{s c}^{t_{k} \hbar}+\tau_{c a}^{t_{k} \hbar}+\right.$ $\left.\tau_{c}^{t_{k} \hbar}\right\}(k=0,1,2, \ldots)$. Essentially, $\underline{t}_{k} \hbar(k=0,1,2, \ldots)$ is also the updating instant for the continuous signal at the rear end of the holder.

Based on the analysis of the data transmission circumstance for nonlinear NCS under nonuniform data transmission, the schematic diagram of data updating sequence is shown in Figure 2, following the rear end of the sampler to the rear end of the holder.

As shown in Figure 2, $t_{k} \hbar$ denotes the transmission instant of effective data which satisfies the trigger condition in the generator and represents the data which can affect the controlled plant through control feedback. These effective data are transmitted to the computing unit of the faulttolerant controller through transmission time-delay $\tau_{s c}^{t_{k} \hbar}$. Afterwards, the multiobjective constraint fault-tolerant controller based on T-S fuzzy model is calculated after computing time-delay $\tau_{c}^{t_{k} \hbar}$. Last, the related control law is conveyed to the zero-order holder after the time-delay $\tau_{c a}^{t_{k} \hbar}$.

2.3. The Establishment of T-S Hybrid Closed Failure NCS Model under Nonuniform Transmission. Suppose that the network transmission time-delay exists in both the front and rear end of the controller and the system state is completely measurable; then the system adopts the static state feedback controller. When $t \in\left[t_{k} \hbar+\tau_{t_{k} \hbar}, t_{k+1} \hbar+\tau_{t_{k+1}} \hbar\right)$, the control signals at the rear end of the T-S fuzzy controller and the front end of the zero-order holder are expressed in (7) and (8), respectively.

$$
\begin{aligned}
& \bar{u}(t)=\sum_{j=1}^{r} h_{j}\left(\theta\left(t_{k} \hbar\right)\right) K_{j} x\left(t_{k} \hbar\right), \quad k=0,1,2, \ldots, t \in\left[t_{k} \hbar+\tau_{s c}^{t_{k} \hbar}+\tau_{c}^{t_{k} \hbar}, t_{k+1} \hbar+\tau_{s c}^{t_{k+1} \hbar}+\tau_{c}^{t_{k+1} \hbar}\right), \\
& u(t)=\sum_{j=1}^{r} h_{j}\left(\theta\left(t_{k} \hbar\right)\right) K_{j} x\left(t_{k} \hbar\right), \quad k=0,1,2, \ldots, t \in\left[t_{k} \hbar+\tau_{s c}^{t_{k} \hbar}+\tau_{c}^{t_{k} \hbar}+\tau_{c a}^{t_{k} \hbar}, t_{k+1} \hbar+\tau_{s c}^{t_{k+1} \hbar}+\tau_{c}^{t_{k+1} \hbar}+\tau_{c a}^{t_{k+1} \hbar}\right),
\end{aligned}
$$

where $K_{j}(j=1,2, \ldots, r)$ is the related state feedback gain matrix. Obviously, $\bigcup_{k=1}^{\infty}\left[t_{k}, t_{k+1}\right)=[0, \infty), h_{k}$ denotes the data transmission period in the event generator, and $h_{k}=$ $t_{k+1} \hbar-t_{k} \hbar$.

Through the preceding analysis, a nonuniform transmission period for the transmission data after the event generator exists even if the sample period in the sampler is uniform. At present, the sampler and generator are regarded as a virtual nonuniform sampler. The updating period in the front end of holder for the traditional nonuniform sample system is determined by the variable sample period of the sampler, whereas the data updating period at the front end of the holder in the present study is determined by the nonuniform transmission period, network transmission time-delay, and computing time-delay for the nonuniform transmission system. Although different factors determine the data updating period on the holder side, the uncertainty of the updating period, which exists in the rear end of the holder in these two kinds of system, is a consistent fact on the macrolevel. Therefore, the study ideas based on traditional nonuniform sample control system are presented when establishing the closed-loop failure model for nonuniform transmission nonlinear NCS under DETCS.

When $x\left(t_{k} \hbar\right)$ has reached the actuator but $x\left(t_{k+1} \hbar\right)$ has not arrived, the transmission interval is defined as

$$
\Omega=\left[t_{k} \hbar+\tau_{t_{k} \hbar}, t_{k+1} \hbar+\tau_{t_{k+1} \hbar}\right) \text {. }
$$

The transmission interval is divided into several subintervals, such that

$$
\Omega=\Delta_{k}^{0} \cup \cdots \Delta_{k}^{1_{k}} \cup \cdots \cup \Delta_{k}^{d_{k}},
$$

where $\Delta_{k}^{l_{k}}=\left[t_{k} \hbar+l_{k} \hbar+\tau_{t_{k} \hbar+l_{k} \hbar}, t_{k} \hbar+\left(l_{k}+1\right) \hbar+\tau_{t_{k} \hbar+\left(l_{k}+1\right) \hbar}\right)$ and $l_{k}=0,1,2, \ldots, d_{k}, d_{k} \hbar=t_{k+1} \hbar-t_{k} \hbar-\hbar . \tau_{t_{k} \hbar+l_{k} \hbar}$ is assumed as 


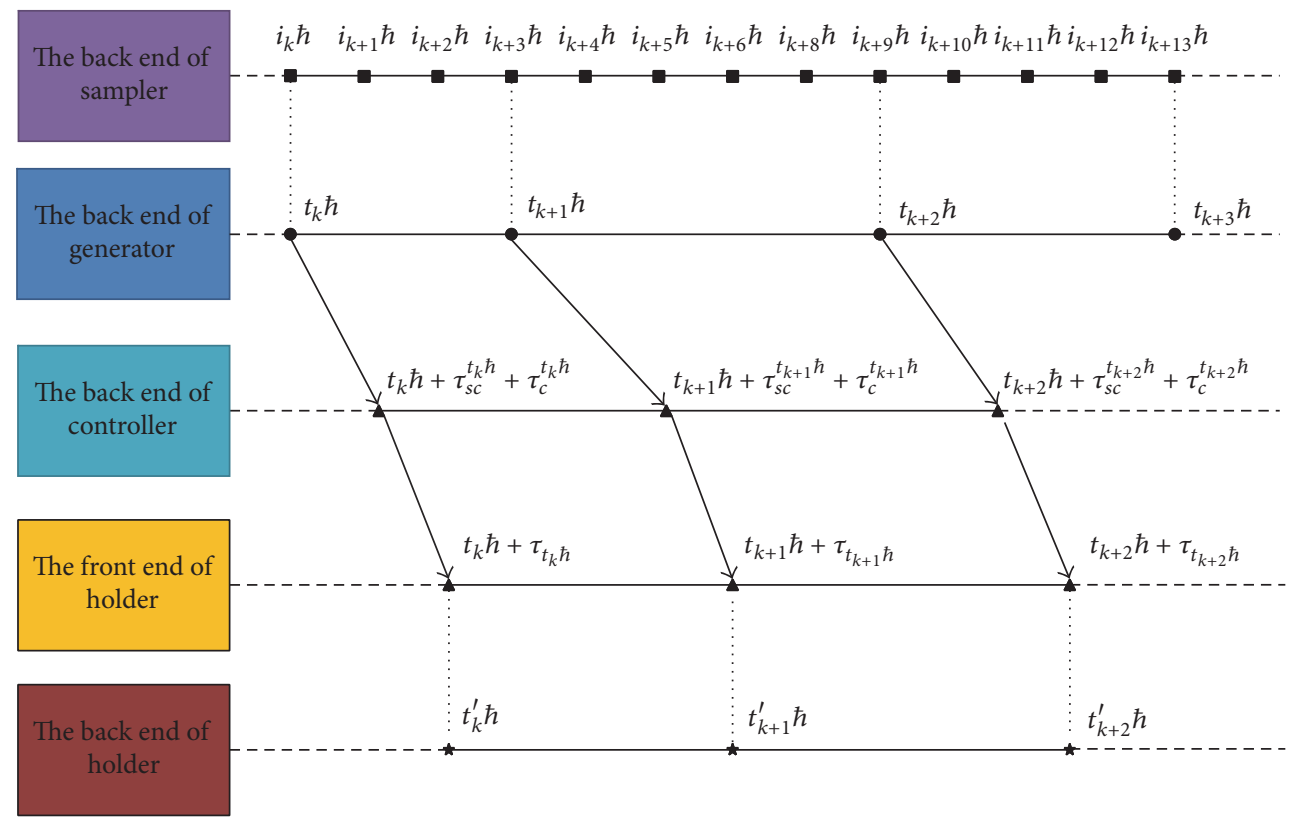

FIGURE 2: The schematic diagram of the effective transmission sequence for the sample signal under DETCS.

the virtual network transmission delay at the sample instant $t_{k} \hbar+l_{k} \hbar$ to guarantee the effectiveness of the interval division.

The static state feedback control law is adopted in the present study. Therefore, three kinds of time-delay $\left(\tau_{s c}^{t_{k} \hbar}, \tau_{c}^{t_{k} \hbar}, \tau_{c a}^{t_{k} \hbar}\right)$ are considered as a kind of comprehensive time-delay $(\tau(t))$. Considering these kinds of time-delay, respectively, is unnecessary. When $t \in \Delta_{k}^{l_{k}}$, the function $\tau(t)$ is defined as

$$
\tau(t)=t-t_{k} \hbar
$$

We define $i_{k}=i_{k} \hbar, t_{k}=t_{k} \hbar, l_{k}=l_{k} \hbar, d_{k}=d_{k} \hbar$ for convenience in the discussion.

Based on (9) and (10), the upper and lower bounds of $\tau(t)$ are described as

$$
\begin{aligned}
0 & <l_{k}+\tau_{t_{k}+l_{k}} \leq \tau(t) \\
& \leq\left(l_{k}+1\right)+\tau_{t_{k}+\left(l_{k}+1\right)} \\
& \leq\left(d_{k}+1\right)+\tau_{t_{k}+\left(l_{k}+1\right)}, \\
\left(d_{k}+1\right)+\tau_{t_{k}+\left(l_{k}+1\right)} & =t_{k+1}-t_{k}+\tau_{t_{k}+\left(l_{k}+1\right)}<h_{\max k}+\bar{\tau} \\
& \leq h_{\tau},
\end{aligned}
$$

where $h_{\tau}=h_{\max k}+\max \left\{\tau_{t_{k}+\left(l_{k}+1\right)}\right\}=h_{\max k}+\bar{\tau} . h_{\tau}$ is the upper bound of the time-delay function in the interval $\Delta_{k}^{l_{k}} \cdot h_{\max k}$ is the upper bound of the variable transmission period which is induced by the event generator. $\bar{\tau}$ is the upper bound of the time-delay $\tau(t)$ at the instant $t_{k+\left(l_{k}+1\right)}$.

Remark 1. Based on (12), the upper and lower bound for the time-delay function are not only related to the upper bound $\bar{\tau}$ of the network transmission time-delay but are also associated with the upper bound $h_{\max k}$ of the variable transmission period which is induced by the discrete event generator. The time-delay analysis method unifies nonuniform transmission period and network time-delay into a unified framework, thereby providing a solid foundation for consecutive model establishment and codesign between fault-tolerance and communication.

When $t \in \Delta_{k}^{l_{k}}$, the state error $e\left(i_{k}\right)$ is defined as

$$
e\left(i_{k}\right)=x\left(i_{k}\right)-x\left(t_{k}\right)
$$

When $t \in \Delta_{k}^{l_{k}}$, based on the combinations of (6), (11), and (13), we obtain

$$
e^{T}\left(i_{k}\right) \Phi e\left(i_{k}\right) \leq \sigma x^{T}(t-\tau(t)) \Phi x(t-\tau(t)) .
$$

Based on the combination of (8) and (11), $u(t)$ is also written as

$$
\begin{aligned}
u(t) & =\sum_{j=1}^{r} h_{j}\left(\theta\left(t_{k}\right)\right) K_{j}\left(x\left(t_{k}\right)\right) \\
& =\sum_{j=1}^{r} h_{j}(\theta(t-\tau(t))) K_{j}(x(t-\tau(t))) .
\end{aligned}
$$

With regard to the model of general actuator failures in [17], the control input with actuator failure is described as

$$
u^{f}(t)=\operatorname{Lu}(t)
$$

Matrix $L$ denotes the mode set of the system actuator failures and describes the fault extent, where $L=\operatorname{diag}\left\{l_{1}, \ldots, l_{m}\right\}$, $l_{q} \in[0,1], q=1,2, \ldots, m ; l_{q}=0$ indicates that the $q$ th system actuator is invalid; $l_{q} \in(0,1)$ implies that the $q$ th system actuator is at fault to some extent; and $l_{q}=1$ denotes 
that the qth system actuator properly operates. Notably, the case of $L=0$ does not exist because the fault-tolerant control technology is based on redundancy.

With regard to (4), (13), (15), and (16), the closed-loop failure system model of the event-triggered nonuniform transmission is obtained for nonlinear NCS with actuator saturation based on the DETCS, which is as follows:

$$
\begin{aligned}
\dot{x}(t) & =\sum_{i=1}^{r} \sum_{j=1}^{r} h_{i}(\theta(t)) h_{j}(\theta(t-\tau(t))) \\
\cdot & {\left[\left(A_{i}+\Delta A_{i}\right) x(t)\right.} \\
+ & \left(B_{i}+\Delta B_{i}\right) \operatorname{sat}\left(L K_{j}\left(x\left(i_{k}\right)-e\left(i_{k}\right)\right)\right) \\
+ & \left.B_{w i} w(t)\right], \\
z_{1}(t) & =\sum_{i=1}^{r} h_{i}(\theta(t)) C_{i 1} x(t), \\
z_{2}(t) & =\sum_{i=1}^{r} h_{i}(\theta(t)) C_{i 2} x(t) .
\end{aligned}
$$

2.4. The Goal of Fault-Tolerant Control under Multiobjective Constraint. Based on DETCS, the goal of codesigning between multiobjective constraint fault-tolerant control and network communication for nonuniform transmission nonlinear NCS with regard to the constraint of actuator saturation and actuator failures is to seek the state feedback controller gain $K$ and the event-triggered weight matrix $\Phi$. $K$ and $\Phi$ can guarantee that the failure nonlinear NCS with actuator saturation satisfies the following conditions:

(1) When $w(t)=0$, the failure nonlinear NCS possesses $\alpha$-safety degree [25].

(2) Under zero initial condition, for any nonzero $w(t) \in L_{2}[0, \infty]$, the failure nonlinear NCS satisfies $\left\|z_{1}(t)\right\|_{2} \leq \gamma_{1}\|w(t)\|_{2}$, where $\gamma_{1}$ is a given scalar and $\|\cdot\|_{2}$ denotes $L_{2}[0, \infty]$ norm. The scalar $\gamma_{1}$ is the disturbance rejection level.

(3) Under the zero initial condition, for any nonzero $w(t) \in L_{2}[0, \infty]$, the system satisfies $\left\|z_{2}(t)\right\|_{\infty} \leq$ $\gamma_{2}\|w(t)\|_{2}$, where $\gamma_{2}$ is a given scalar and $\|\cdot\|_{2}$ denotes $L_{2}[0, \infty]$ norm. The constraint index can guarantee that the output peak will be smaller than a certain value. The scalar $\gamma_{2}$ can also be seen as the output peak rejection level.

(4) Based on the hypothesis of satisfying the preceding constraint indexes and all the indexes possessing compatibility, the codesign method guarantees a possibly less occupancy rate of the network communication resource.

2.5. Lemma Preparation. In this paper, we used three definitions which are in our previous paper [25]: $\alpha$-safety degree, attraction domain of fault-tolerance with $\alpha$-safety degree, and the contractively invariant set of fault-tolerance with $\alpha$-safety degree.
$\ell(F)=\left\{x_{0} \in R^{n}:\left|f_{l} x\right| \leq 1, l=1,2, \ldots, m\right\}$, where matrix $F \in R^{m \times n}$, and $f_{l}$ denotes the $l$ th row of the matrix $F$; thereafter, $\ell(F)$ is defined as the region where the feedback control $u=\operatorname{sat}(F x)$ is linear for $x$, as indicated in [26].

Based on an ellipsoid estimation of the attraction domain, $P \in R^{n \times n}$ is a positive definite matrix. For $\rho>0$, the ellipsoid is defined as $\varepsilon(P, \rho)=\left\{x \in R^{n}, x^{T} P x \leq \rho\right\}$, where $\varepsilon(P)$ denotes $\varepsilon(P, 1)$.

Lemma 2 (reciprocal convex inequality [27]). For any matrix $M=\left[\begin{array}{ll}R & U \\ * & R\end{array}\right] \geq 0$, scalars $0 \leq d(t) \leq d$, and a vector function $\dot{x}:[-d, 0] \rightarrow \mathbb{R}^{n}$ such that the integration in the following inequality is well defined, thereby holding

$$
-d \int_{t-d}^{t} \dot{x}^{T}(\alpha) R \dot{x}(\alpha) d \alpha \leq \vartheta^{T}(t) Z \vartheta(t),
$$

where

$$
\begin{aligned}
\vartheta^{T}(t) & =\left[\begin{array}{lcc}
x^{T}(t) & x^{T}(t-d(t)) & x^{T}(t-d)
\end{array}\right], \\
Z & =\left[\begin{array}{ccc}
-R & R-U & U \\
* & -2 R+U+U^{T} & R-U \\
* & * & -R
\end{array}\right] .
\end{aligned}
$$

Lemma 3 (see [1]). Let $Y>0$, and $\omega(s)$ is an appropriate dimensional vector. Thereafter, we have the following:

$$
\begin{aligned}
-\int_{t_{1}}^{t_{2}} \omega^{T}(s) Y \omega(s) d s \leq & \left(t_{2}-t_{1}\right) \xi_{t}^{T} F^{T} Y^{-1} F \xi_{t} \\
& +2 \xi_{t}^{T} F^{T} \int_{t_{1}}^{t_{2}} \omega(s) d s
\end{aligned}
$$

where matrix $F$ and vector $\xi_{t}$, which are independent on the integral variable, are arbitrary appropriate dimensional ones.

Lemma 4 (linear convex combination [28]). For any matrices $Z_{i}(i=1,2,3)$ with proper dimensions, then

$$
Z_{1}+\delta Z_{2}+(1-\delta) Z_{3}<0
$$

holds for $\forall \delta \in[0,1]$ if and only if the following set of inequalities hold:

$$
\begin{aligned}
& Z_{1}+Z_{2}<0 \\
& Z_{1}+Z_{3}<0 .
\end{aligned}
$$

Lemma 5 (Wirtinger's inequality [29]). Let $z(t) \in W[a, b)$ which denotes the space of functions $\Phi:[a, b] \rightarrow \mathbb{R}^{n}$, which are absolutely continuous on $[a, b)$, have a finite $\lim _{\theta \rightarrow b^{-}} \Phi(\theta)$, and have square integrable first-order derivatives with the norm $\|\Phi\|_{W}=\max _{\theta \in[a, b]}|\Phi(\theta)|+\left[\int_{a}^{b}|\dot{\Phi}(s)|^{2} d s\right]^{1 / 2}$. Furthermore, if $z(a)=0$, then for any $n \times n$ matrix $R>0$, the following inequality holds: $\pi^{2} \int_{a}^{b} z^{T}(s) R z(s) d s \leq \int_{a}^{b} \dot{z}^{T}(s) R \dot{z}(s) d s$.

\section{Main Results}

3.1. Condition of the Invariant Set. Two cases for the system with different performances are studied as follows. 
3.1.1. The Sufficient Condition of the Invariant Set for the Closed-Loop Failure System with $\alpha$-Safety Degree

Theorem 6. Based on the event-triggered condition (6) of the DETCS, there exist some matrices $P=P^{T}>0, Q=Q^{T}>0$, $R=R^{T}>0, S=S^{T}>0, T=T^{T}>0, W=W^{T}>$ $0, U$, and $V$ for the given values $\sigma, h_{\tau}$, and $\alpha$, the known matrices $K_{j}(j=1,2, \ldots, r)$, and $\Phi$. If all the given values and the matrix variables satisfy $\varepsilon(P) \subset \ell(F)$ and the matrix inequalities

$$
\begin{gathered}
{\left[\begin{array}{ll}
R & U \\
* & R
\end{array}\right] \geq 0,} \\
{\left[\begin{array}{cc}
\Pi^{0 i j} & h_{\tau} \widehat{V} \\
* & -h_{\tau} S
\end{array}\right]<0,} \\
{\left[\begin{array}{cc}
\Pi^{0 i j} & h_{\tau} \Lambda_{0}^{i j} \\
* & -h_{\tau} S^{-1}
\end{array}\right]<0}
\end{gathered}
$$

then system (17) remains asymptotically stable in the domain of attraction $\varepsilon(P)$ and possesses $\alpha$-safety degree. That is, (15) is a nonuniform transmission robust fault-tolerant control law which can enable the system (17) to possess $\alpha$-safety degree and a lower occupancy rate of network resource, where

$$
\begin{aligned}
\Pi_{11}^{0 i j}= & P \bar{A}+\bar{A}^{T} P+Q-R+V_{1}+V_{1}^{T}-\frac{\pi^{2}}{4} W, \\
\Pi_{12}^{0 i j}= & P \bar{B}\left\{\Upsilon_{q} K_{j}+\Upsilon_{q}^{-} F_{j}\right\}+R-U-V_{1}+V_{2}^{T} \\
& +\frac{\pi^{2}}{4} W, \\
\Pi_{13}^{0 i j}= & U+V_{3}^{T}, \\
\Pi_{15}^{0 i j}= & h_{\tau} \bar{A}^{T}, \\
\Pi_{22}^{0 i j}= & -2 R+U+U^{T}-V_{2}-V_{2}^{T}+h_{\tau} T-\frac{\pi^{2}}{4} W \\
& +\sigma \exp (2 \alpha \tau(t)) \Phi,
\end{aligned}
$$

$$
\begin{aligned}
& V_{1}(\eta(t))=\eta^{T}(t) P \eta(t)+\int_{t-h_{\tau}}^{t} \eta^{T}(s) Q \eta(s) d s, \\
& V_{2}(\eta(t))=h_{\tau} \int_{-h_{\tau}}^{0} \int_{t+s}^{t} \dot{\eta}^{T}(\delta) R \dot{\eta}(\delta) d \delta d s, \\
& V_{3}(\eta(t))=\left(h_{\tau}-\tau(t)\right) \int_{t-\tau(t)}^{t} \dot{\eta}^{T}(\delta) S \dot{\eta}(\delta) d \delta, \\
& V_{4}(\eta(t))=\left(h_{\tau}-\tau(t)\right) \tau(t) \eta^{T}(t-\tau(t)) T \eta(t-\tau(t)),
\end{aligned}
$$

$$
\begin{aligned}
& \Pi_{23}^{0 i j}=R-U-V_{3}^{T}, \\
& \Pi_{25}^{0 i j}=h_{\tau}\left\{\Upsilon_{q} K_{j}+\Upsilon_{q}^{-} F_{j}\right\}^{T} \bar{B}^{T}, \\
& \Pi_{33}^{0 i j}=-Q-R, \\
& \Pi_{44}^{0 i j}=-\Phi, \\
& \Pi_{55}^{0 i j}=-(R+W)^{-1}, \\
& \Lambda_{0}^{i j T}=\left[\begin{array}{llllll}
\bar{A} & \bar{B}\left\{\Upsilon_{q} K_{j}+\Upsilon_{q}^{-} F_{j}\right\} & 0 & 0 & 0
\end{array}\right], \\
& \widehat{V}^{T}=\left[\begin{array}{lllll}
V_{1}^{T} & V_{2}^{T} & V_{3}^{T} & 0 & 0
\end{array}\right] .
\end{aligned}
$$

Proof. The state transition $x(t)=\exp (-\alpha t) \eta(t)$ must be introduced into the proof course for system (17) to obtain $\alpha$ safety degree. Based on Lemma 5 [25], when $\varepsilon(P) \subset \ell(F)$ and $w(t)=0$, then

$$
\begin{aligned}
& \dot{\eta}(t)=\sum_{i=1}^{r} \sum_{j=1}^{r} h_{i}(\theta(t)) h_{j}(\theta(t-\tau(t))) \\
& \cdot\left[\bar{A} \eta(t)+\bar{B} c o\left\{\Upsilon_{q} K_{j}+\Upsilon_{q}^{-} F_{j}\right\} \eta(t-\tau(t))\right], \\
& z_{\alpha 1}(t)=\sum_{i=1}^{r} h_{i}(\theta(t)) C_{i 1} \eta(t), \\
& z_{\alpha 2}(t)=\sum_{i=1}^{r} h_{i}(\theta(t)) C_{i 2} \eta(t),
\end{aligned}
$$

where $\bar{A}=A_{i}+\Delta A_{i}+\alpha I, \bar{B}=\exp (\alpha \tau(t))\left(B_{i}+\Delta B_{i}\right) L, z_{\alpha 1}(t)=$ $\exp (\alpha t) z_{1}(t)$, and $z_{\alpha 2}(t)=\exp (\alpha t) z_{2}(t)$.

Based on definition 2 of [25], if system (27) is asymptotically stable, thereafter, system (17) will possess $\alpha$-safety degree.

A discontinuous Lyapunov-Krasovskii functional candidate is constructed to reduce the conservatism and capture the sampling variable characteristic of a saw tooth time-delay $\dot{\tau}(t)=1, t \in\left(t_{k}, t_{k+1}\right), k \in N$ as follows:

$$
\begin{aligned}
V(\eta(t))= & V_{1}(\eta(t))+V_{2}(\eta(t))+V_{3}(\eta(t)) \\
& +V_{4}(\eta(t))+V_{5}(\eta(t)),
\end{aligned}
$$

where 


$$
\begin{array}{r}
V_{5}(\eta(t))=h_{\tau}^{2} \int_{t-\tau(t)}^{t} \dot{\eta}^{T}(\delta) W \dot{\eta}(\delta) d \delta-\frac{\pi^{2}}{4} \int_{t-\tau(t)}^{t}[\eta(\delta)-\eta(t-\tau(t))]^{T} W[\eta(\delta)-\eta(t-\tau(t))] d \delta, \\
P=P^{T}>0, Q=Q^{T}>0, R=R^{T}>0, S=S^{T}>0, T=T^{T}>0, W=W^{T}>0 .
\end{array}
$$

It is not difficult to understand that $\widehat{V}(\eta(t))=\left(h_{\tau}-\right.$ $\tau(t)) \int_{t-\tau(t)}^{t} \dot{\eta}^{T}(\delta) S \dot{\eta}(\delta) d \delta$ is discontinuous at the transmission instants $t_{k}, k \in N . \widehat{V}(\eta(t))$ is nonnegative only before the jumps $t_{k}$ and vanishes just after the jumps, such as $\widehat{V}\left(\eta\left(t_{k}^{-}\right)\right) \geq$ $0, \widehat{V}\left(\eta\left(t_{k}^{+}\right)\right)=0 . \widetilde{V}(\eta(t))=\left(h_{\tau}-\tau(t)\right) \tau(t) \eta^{T}(t-\tau(t)) T \eta(t-\tau(t))$ is continuous on $[0, \infty)$ and $\widetilde{V}\left(\eta\left(t_{k}\right)\right)=0$. It is easy to verify that $\widehat{V}(\eta(t))=h_{\tau}^{2} \int_{t-\tau(t)}^{t} \dot{\eta}^{T}(\delta) W \dot{\eta}(\delta) d \delta-\left(\pi^{2} / 4\right) \int_{t-\tau(t)}^{t}[\eta(\delta)-$ $\eta(t-\tau(t))]^{T} W[\eta(\delta)-\eta(t-\tau(t))] d \delta \geq 0$ from Lemma 5 . Furthermore, it is correct that $\widehat{V}\left(\eta\left(t_{k}^{-}\right)\right) \geq 0, \widehat{V}\left(\eta\left(t_{k}^{+}\right)\right)=0$. Therefore, $V\left(\eta\left(t_{k}^{-}\right)\right) \geq V\left(\eta\left(t_{k}^{+}\right)\right)$is concluded.

For $t \in\left(t_{k}, t_{k+1}\right), k \in N$, taking the derivative of $V(t)$ along the trajectory of (27), we can obtain

$$
\begin{aligned}
& \dot{V}(\eta(t)) \leq \max _{q \in\left[1,2, \ldots, 2^{m}\right]} \sum_{i=1}^{r} \sum_{j=1}^{r} h_{i}(\theta(t)) h_{j}(\theta(t-\tau(t))) \\
& \cdot\left[\dot{V}_{1}(\eta(t))+\dot{V}_{2}(\eta(t))+\dot{V}_{3}(\eta(t))+\dot{V}_{4}(\eta(t))+\dot{V}_{5}(\eta(t))\right] \\
& +e_{\alpha}^{T}\left(i_{k}\right) \Phi e_{\alpha}\left(i_{k}\right)-e_{\alpha}^{T}\left(i_{k}\right) \Phi e_{\alpha}\left(i_{k}\right),
\end{aligned}
$$

where

$$
\begin{aligned}
& \dot{V}_{1}(\eta(t))=2 \eta^{T}(t) P \bar{A} \eta(t)+2 \eta^{T}(t) P \bar{B}\left\{\gamma_{q} K_{j}\right. \\
& \left.+\gamma_{q}^{-} F_{j}\right\} \eta(t-\tau(t))+\eta^{T}(t) Q \eta(t)-\eta^{T}\left(t-h_{\tau}\right) \\
& \cdot Q \eta\left(t-h_{\tau}\right), \\
& \dot{V}_{2}(\eta(t))=h_{\tau}^{2} \dot{\eta}^{T}(t) R \dot{\eta}(t)-h_{\tau} \int_{t-h_{\tau}}^{t} \dot{\eta}^{T}(s) R \dot{\eta}(s) d s, \\
& \dot{V}_{3}(\eta(t))=-\int_{t-\tau(t)}^{t} \dot{\eta}^{T}(\delta) \operatorname{Si}(\delta) d \delta \\
& +\left(h_{\tau}-\tau(t)\right) \dot{\eta}^{T}(t) \operatorname{Si}(t), \\
& \dot{V}_{4}(\eta(t))=h_{\tau} \eta^{T}(t-\tau(t)) T \eta(t-\tau(t))+2\left(h_{\tau}\right. \\
& \quad-\tau(t)) \eta^{T}(t-\tau(t)) T \eta(t-\tau(t)), \\
& \dot{V}_{5}(\eta(t))=h_{\tau}^{2} \dot{\eta}^{T}(t) W \dot{\eta}(t)-\frac{\pi^{2}}{4}[\eta(t) \\
& \quad-\eta(t-\tau(t))]^{T} W[\eta(t)-\eta(t-\tau(t))] .
\end{aligned}
$$

Based on (27), we can obtain the quadratic term of the state derivative, which is estimated as follows:

$$
\begin{aligned}
& h_{\tau}^{2} \dot{\eta}^{T}(t) R \dot{\eta}(t)+h_{\tau}^{2} \dot{\eta}^{T}(t) W \dot{\eta}(t) \\
& \leq \max _{q \in\left[1,2, \ldots, 2^{m}\right]} \sum_{i=1}^{r} \sum_{j=1}^{r} h_{i}(\theta(t)) h_{j}(\theta(t-\tau(t))) h_{\tau}^{2} \zeta^{T}(t) \\
& \cdot \Lambda_{1}^{i j}(R+W) \Lambda_{1}^{i j T} \zeta(t),
\end{aligned}
$$

$$
\begin{gathered}
\left(h_{\tau}-\tau(t)\right) \dot{\eta}^{T}(t) S \dot{\eta}(t) \leq \max _{q \in\left[1,2, \ldots, 2^{m}\right]} \sum_{i=1}^{r} \sum_{j=1}^{r} h_{i}(\theta(t)) \\
\cdot h_{j}(\theta(t-\tau(t)))\left(h_{\tau}-\tau(t)\right) \zeta^{T}(t) \Lambda_{1}^{i j} S \Lambda_{1}^{i j T} \zeta(t),
\end{gathered}
$$

where $\zeta^{T}(t)=\left[\eta^{T}(t) \eta^{T}(t-\tau(t)) \eta^{T}\left(t-h_{\tau}\right)\right], \Lambda_{1}^{i j T}=$

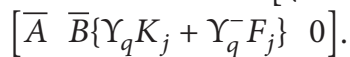

Based on Lemma 2, we can obtain

$$
\begin{aligned}
& -h_{\tau} \int_{t-h_{\tau}}^{t} \dot{\eta}^{T}(s) R \dot{\eta}(s) d s \\
& \leq \zeta^{T}(t)\left[\begin{array}{ccc}
-R & R-U & U \\
* & -2 R+U+U^{T} & R-U \\
* & * & -R
\end{array}\right] \zeta(t) \\
& \text { subject to }\left[\begin{array}{ll}
R & U \\
* & R
\end{array}\right] \geq 0
\end{aligned}
$$

and (34) is equal to (23).

Based on Lemma 3, we have

$$
\begin{aligned}
& -\int_{t-\tau(t)}^{t} \dot{\eta}^{T}(\delta) S \dot{\eta}(\delta) d \delta \\
& \leq \tau(t) \zeta^{T}(t) V^{T} S^{-1} V \zeta(t)+\zeta^{T}(t)\left[\begin{array}{lll}
V & -V & 0
\end{array}\right] \zeta(t) \\
& \quad+\zeta^{T}(t)\left[\begin{array}{lll}
V & -V & 0
\end{array}\right]^{T} \zeta(t),
\end{aligned}
$$

where $V^{T}=\left[\begin{array}{lll}V_{1}^{T} & V_{2}^{T} & V_{3}^{T}\end{array}\right]$.

When $t \in\left(t_{k}, t_{k+1}\right)$, according to (14) and $e_{\alpha}\left(i_{k}\right)=$ $\exp (\alpha t) e\left(i_{k}\right)$, we have

$$
\begin{aligned}
& e_{\alpha}^{T}\left(i_{k}\right) \Phi e_{\alpha}\left(i_{k}\right) \\
& \quad \leq \sigma \exp (2 \alpha \tau(t)) \eta^{T}(t-\tau(t)) \Phi \eta(t-\tau(t)) .
\end{aligned}
$$

Based on (32)-(36), we have

$$
\begin{array}{r}
\dot{V}(\eta(t)) \leq \max _{q \in\left[1,2, \ldots, 2^{m}\right]} \sum_{i=1}^{r} \sum_{j=1}^{r} h_{i}(\theta(t)) h_{j}(\theta(t-\tau(t))) \\
\cdot\left\{\varsigma^{T}(t)\left[\Pi^{1 i j}+\tau(t) \Omega_{1}^{i j}+\left(h_{\tau}-\tau(t)\right) \Theta_{1}^{i j}\right] \varsigma(t)\right\},
\end{array}
$$


where

$$
\begin{aligned}
\varsigma^{T}(t) & =\left[\begin{array}{ll}
\xi^{T}(t) & e^{T}\left(i_{k}\right)
\end{array}\right], \\
\Pi^{1 i j} & =\Pi^{0 i j}, \\
\Omega_{1}^{i j} & =\widetilde{V} S^{-1} \widetilde{V}^{T}, \\
\Theta_{1}^{i j} & =\Lambda_{2}^{i j} S \Lambda_{2}^{i j T}+2 \Delta_{0} T \Delta_{0}^{T}, \\
\Lambda_{2}^{i j T} & =\left[\begin{array}{llll}
\bar{A} \bar{B}\left\{\Upsilon_{q} K_{j}+\Upsilon_{q}^{-} F_{j}\right\} & 0 & 0
\end{array}\right], \\
\widetilde{V}^{T} & =\left[\begin{array}{llll}
V_{1}^{T} & V_{2}^{T} & V_{3}^{T} & 0
\end{array}\right], \\
\Delta_{0}^{T} & =\left[\begin{array}{llll}
0 & I & 0 & 0
\end{array}\right] .
\end{aligned}
$$

Based on Lemma 4, we have

$$
\begin{aligned}
& \Pi^{1 i j}+h_{\tau} \Omega_{1}^{i j}<0 \\
& \Pi^{1 i j}+h_{\tau} \Theta_{1}^{i j}<0 .
\end{aligned}
$$

Thereafter, we obtain (24) and (25) by applying the Schur Complement Lemma into (39). Therefore, if (23), (24), (25), and $\varepsilon(P) \subset \ell(F)$ are satisfied, then (17) possesses $\alpha$-safety degree. Moreover, the ellipsoid $\varepsilon(P)$ is the invariant set for system (17). In addition, the system possesses less occupancy of the network resource. The proof is hereby completed.

\subsubsection{Sufficient Condition of Invariant Set for Closed-Loop Fail-} ure System with Multiobjective Constraint Performance Index. People also expect that the failure networked fuzzy system possesses several additional performances, such as $\alpha$-safety degree, $H_{\infty}$ performance index, and $H_{2}$ performance index excluding stability in the field of fault-tolerant control. All the indexes are described in Section 2.4 in detail. Therefore, the multiobjective constraint fault-tolerant control problem for nonuniform transmission nonlinear NCS (17) under the DETCS should be studied as follows.

Theorem 7. Based on the event-triggered condition (6) of the DETCS, there exist some matrices $P=P^{T}>0, Q=Q^{T}>0$, $R=R^{T}>0, S=S^{T}>0, T=T^{T}>0, W=W^{T}>0$, $U$ and $V$ for the given values $\sigma, h_{\tau}, \alpha, \gamma_{1}$, and $\gamma_{2}$, the known matrices $K_{j}(j=1,2, \ldots, r)$, and $\Phi$. If all the given values and the matrix variables satisfy $\varepsilon(P) \subset \ell(F)$ and the matrix inequalities

$$
\begin{aligned}
{\left[\begin{array}{ll}
R & U \\
* & R
\end{array}\right] } & \geq 0, \\
{\left[\begin{array}{cc}
\Pi^{2 i j} & h_{\tau} \breve{V} \\
* & -h_{\tau} S
\end{array}\right] } & <0, \\
{\left[\begin{array}{cc}
\Pi^{2 i j} & h_{\tau} \Lambda_{3}^{i j} \\
* & -h_{\tau} S^{-1}
\end{array}\right] } & <0, \\
{\left[\begin{array}{ll}
P & C_{i 2}^{T} \\
* & \gamma_{2}^{2} I
\end{array}\right] } & \geq 0
\end{aligned}
$$

then system (17) remains asymptotically stable in the domain of attraction $\varepsilon(P)$ and possesses $\alpha$-safety degree, $\gamma_{1}$-disturbance rejection level and $\gamma_{2}$-out-peak rejection level. That is, (15) denotes the nonuniform transmission multiobjective constraint robust fault-tolerant control law which can allow system (17) to possess the multiobjective constraint performance and less possible network communication occupancy, where

$$
\begin{aligned}
& \Pi_{11}^{2 i j}=P \bar{A}+\bar{A}^{T} P+Q-R+V_{1}+V_{1}^{T}-\frac{\pi^{2}}{4} W, \\
& \Pi_{12}^{2 i j}=P \bar{B}\left\{\Upsilon_{q} K_{j}+\Upsilon_{q}^{-} F_{j}\right\}+R-U-V_{1}+V_{2}^{T} \\
& +\frac{\pi^{2}}{4} W \\
& \Pi_{13}^{2 i j}=U+V_{3}^{T}, \\
& \Pi_{15}^{2 i j}=P B_{w i}, \\
& \Pi_{16}^{2 i j}=C_{i 1}^{T} \text {, } \\
& \Pi_{17}^{2 i j}=h_{\tau} \bar{A}^{T}, \\
& \Pi_{23}^{2 i j}=R-U-V_{3}^{T} \text {, } \\
& \Pi_{27}^{2 i j}=h_{\tau}\left\{\Upsilon_{q} K_{j}+\Upsilon_{q}^{-} F_{j}\right\}^{T} \bar{B}^{T}, \\
& \Pi_{22}^{2 i j}=-2 R+U+U^{T}-V_{2}-V_{2}^{T}+h_{\tau} T-\frac{\pi^{2}}{4} W \\
& +\sigma \exp (2 \alpha \tau(t)) \Phi, \\
& \Pi_{33}^{2 i j}=-Q-R, \\
& \Pi_{44}^{2 i j}=-\Phi, \\
& \Pi_{55}^{2 i j}=-\gamma_{1}^{2} I, \\
& \Pi_{57}^{2 i j}=h_{\tau} B_{w i}^{T}, \\
& \Pi_{66}^{2 i j}=-e_{i} I \text {, } \\
& \Pi_{77}^{2 i j}=-(R+W)^{-1}, \\
& \widehat{V}=\left[\begin{array}{lllllll}
V_{1} & V_{2} & V_{3} & 0 & 0 & 0 & 0
\end{array}\right]^{T}, \\
& \Lambda_{3}^{i j}=\left[\begin{array}{lllllll}
\bar{A}^{T} & \left\{\Upsilon_{q} K_{j}+\Upsilon_{q}^{-} F_{j}\right\}^{T} \bar{B}^{T} & 0 & 0 & B_{w i}^{T} & 0 & 0
\end{array}\right]^{T} .
\end{aligned}
$$

Proof. Under the zero initial condition and $w(t) \neq 0$, when $t \in\left(t_{k}, t_{k+1}\right)$, the following function of $H_{\infty}$ performance index is considered:

$$
\begin{aligned}
& J_{z_{\alpha 1} w}=\int_{0}^{t}\left(z_{\alpha 1}^{T}(s) z_{a 1}(s)-\gamma_{1}^{2} w^{T}(s) w(s)\right) d s \\
& =\int_{0}^{t}\left(z_{\alpha 1}^{T}(s) z_{a 1}(s)-\gamma_{1}^{2} w^{T}(s) w(s)\right. \\
& \quad+\dot{V}(\eta(s))) d s-V(\eta(t))
\end{aligned}
$$




$$
\begin{aligned}
& \leq \int_{0}^{t}\left(z_{\alpha 1}^{T}(s) z_{a 1}(s)-\gamma_{1}^{2} w^{T}(s) w(s)\right. \\
& +\dot{V}(\eta(s))) d s .
\end{aligned}
$$

Similar to the proof procedure of Theorem 6 , if we consider the uncertain disturbance $(w(t) \neq 0)$, we can obtain

$$
\begin{aligned}
& \dot{V}(\eta(t)) \leq \max _{q \in\left[1,2, \ldots, 2^{m}\right]} \sum_{i=1}^{r} \sum_{j=1}^{r} h_{i}(\theta(t)) h_{j}(\theta(t-\tau(t))) \\
& \cdot\left\{\zeta^{T}(t)\left[\Pi^{3 i j}+\tau(t) \Omega_{2}^{i j}+\left(h_{\tau}-\tau(t)\right) \Theta_{2}^{i j}\right] \zeta(t)\right\} \\
& \quad-z_{\alpha 1}^{T}(t) z_{\alpha 1}(t)+\gamma_{1}^{2} w^{T}(t) w(t),
\end{aligned}
$$

where

$$
\begin{aligned}
& \zeta^{T}(t)=\left[\begin{array}{lll}
\xi^{T}(t) & e^{T}\left(i_{k}\right) & w^{T}(t)
\end{array}\right], \\
& \Pi^{3 i j}=\Pi^{2 i j}, \\
& \Omega_{2}^{i j}=\widehat{V} S^{-1} \widehat{V}^{T} \\
& \Theta_{2}^{i j}=\Lambda_{4}^{i j} S \Lambda_{4}^{i j T}+2 \Delta_{1} T \Delta_{1}^{T},
\end{aligned}
$$

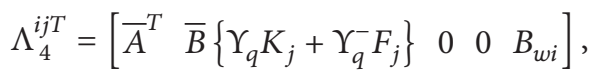

$$
\begin{aligned}
& \widehat{V}^{T}=\left[\begin{array}{lllll}
V_{1}^{T} & V_{2}^{T} & V_{3}^{T} & 0 & 0
\end{array}\right], \\
& \Delta_{1}^{T}=\left[\begin{array}{lllll}
0 & I & 0 & 0 & 0
\end{array}\right] .
\end{aligned}
$$

If $\Pi^{3 i j}+\tau(t) \Omega_{2}^{i j}+\left(h_{\tau}-\tau(t)\right) \Theta_{2}^{i j}<0$, then $z_{\alpha 1}^{T}(t) z_{a 1}(t)-$ $\gamma_{1}^{2} w^{T}(t) w(s)+\dot{V}(\eta(t))<0$.

Combining $\Pi^{3 i j}+\tau(t) \Omega_{2}^{i j}+\left(h_{\tau}-\tau(t)\right) \Theta_{2}^{i j}$ with Lemma 4 , we can obtain

$$
\begin{aligned}
& \Pi^{3 i j}+h_{\tau} \Omega_{2}^{i j}<0 \\
& \Pi^{3 i j}+h_{\tau} \Theta_{2}^{i j}<0
\end{aligned}
$$

Combining (48) with Schur Complement, we can obtain (41) and (42).

Similarly, suppose the zero initial condition; then $\left.V(\eta(t))\right|_{t=0}=0$. The following generalized function of $\mathrm{H}_{2}$ performance index is considered:

$$
J_{z_{\alpha 2} w}=V(\eta(t))-\int_{0}^{t} w^{T}(s) w(s) d s .
$$

For any nonzero $w(t) \in L_{2}[0, \infty)$ and $t \geq 0$, we can obtain

$$
J_{z_{\alpha 2} w}=V(\eta(t))-\left.V(\eta(t))\right|_{t=0}-\int_{0}^{t} w^{T}(s) w(s) d s .
$$

From the definition of Lyapunov function and the expression of $J_{z_{\alpha 2} w}$, we can obtain

$$
\eta^{T}(t) P \eta(t) \leq V(\eta(t)) \leq \int_{0}^{t} w^{T}(s) w(s) d s .
$$

Combining (43) with Schur Complement, we can obtain

$$
\left[\begin{array}{cc}
P & C_{i 2}^{T} \\
* & \gamma_{2}^{2} I
\end{array}\right] \geq 0 \Longleftrightarrow P-\left(\frac{1}{\gamma_{2}^{2}}\right) C_{i 2}^{T} C_{i 2}>0 \Longleftrightarrow C_{i 2}^{T} C_{i 2}<\gamma_{2}^{2} P
$$

Based on (27), (51), and (52), we can obtain

$$
\begin{aligned}
z_{\alpha 2}^{T}(t) z_{\alpha 2}(t) & =\eta^{T}(t) C_{i 2}^{T} C_{i 2} \eta(t)<\gamma_{2}^{2} \eta^{T}(t) P \eta(t) \\
& \leq \gamma_{2}^{2} \int_{0}^{t} w^{T}(s) w(s) d s \\
& \leq \gamma_{2}^{2} \int_{0}^{\infty} w^{T}(s) w(s) d s
\end{aligned}
$$

From (53), for any $w(t) \in L_{2}[0, \infty)$ and $t>0$, the generalized $H_{2}$ performance index $\left\|z_{\alpha 2}(t)\right\|_{\infty} \leq \gamma_{2}\|w(t)\|_{2}$ is satisfied; that is, system (17) has an $\mathrm{H}_{2}$ performance index.

Therefore, if matrix inequalities (40)-(43) are simultaneously satisfied, system (17) possesses $\alpha$-safety degree, $\left\|z_{\alpha 1}(t)\right\|_{2} \leq \gamma_{1}\|w(t)\|_{2},\left\|z_{\alpha 2}(t)\right\|_{\infty} \leq \gamma_{2}\|w(t)\|_{2}$, and fewer transmission data. The ellipsoid $\varepsilon(P)$ is the invariant set of system (17). Particularly, the feedback control law (15) can make the nonuniform transmission nonlinear NCS (17) remain inside the domain of attraction $\varepsilon(P)$ and possess some multiobjective constraint performances index under the DETCS. The proof is completed.

3.2. The Codesign Method. The codesign in this paper is between the multiobjective constraint fault-tolerant control and the network communication for system (17) with nonuniform transmission period. Codesign refers to simultaneously obtaining the multiobjective constraint fault-tolerant controller and the event-triggered weight matrix by solving a set of linear matrix inequalities. However, only by providing the controller in advance can we obtain the event-triggered weight matrix in the previous study result [31]. Simultaneously obtaining the controller and the event-triggered weight matrix is emphasized in the proposed codesign method in the present study. The balance between the quality of control for the system and the quality of service for the network can be obtained by adjusting the related parameter.

3.2.1. The Codesign Method between Fault-Tolerance and Communication for Closed-Loop Failure System with $\alpha$-Safety Degree. The goal of system codesign is to obtain the appropriate fault-tolerant control gain and event-triggered weight matrix for system (17), thereby making the system asymptotically stable and obtaining other performance indexes such as $\alpha$-stability and less occupancy of network communication resource. 
Theorem 8. Based on the event-triggered condition (6) of the DETCS, there exist some matrices $X=X^{T}>0, \bar{\Phi}=\bar{\Phi}^{T}>0$, $\bar{Q}, \bar{R}, \bar{S}, \bar{T}, \bar{W}, \bar{K}_{j}(j=1,2, \ldots, r), \bar{U}$ and $\bar{V}$ for the given values $\sigma, h_{\tau}$, and $\alpha$. If all the given values and the matrix variables satisfy $\varepsilon(P) \subset \ell(F)$ and the matrix inequalities

$$
\begin{aligned}
& {\left[\begin{array}{ll}
\bar{R} & \bar{U} \\
* & \bar{R}
\end{array}\right] \geq 0,} \\
& {\left[\begin{array}{ccc}
\Pi^{4 i j} & h_{\tau} \bar{V} & \Gamma_{0}^{i j} \\
* & -h_{\tau} \bar{S} & 0 \\
* & * & \Xi_{0}^{i j}
\end{array}\right]<0,} \\
& {\left[\begin{array}{ccc}
\Pi^{4 i j} & h_{\tau} \Lambda_{5}^{i j} & \widehat{\Gamma}_{0}^{i j} \\
* & h_{\tau} \bar{S}-2 h_{\tau} X & 0 \\
* & * & \Xi_{0}^{i j}
\end{array}\right]<0,} \\
& {\left[\begin{array}{ll}
1 & \widetilde{f}_{l} \\
* & X
\end{array}\right] \geq 0, \quad l \in[1, m],} \\
& \Pi_{11}^{4 i j} \\
& =X\left(A_{i}+\alpha I\right)+\left(A_{i}+\alpha I\right)^{T} X+\bar{Q}-\bar{R}+\bar{V}_{1} \\
& +\bar{V}_{1}^{T}-\frac{\pi^{2}}{4} \bar{W}
\end{aligned}
$$$$
\Pi_{12}^{4 i j}
$$$$
=\exp (\alpha \tau(t)) P B_{i} L\left\{\Upsilon_{q} \bar{K}_{j}+\Upsilon_{q}^{-} \bar{F}_{j}\right\}+\bar{R}-\bar{U}-\bar{V}_{1}
$$$$
+\bar{V}_{2}^{T}+\frac{\pi^{2}}{4} \bar{W}
$$$$
\Pi_{13}^{4 i j}=\bar{U}+\bar{V}_{3}^{T}
$$$$
\Pi_{15}^{4 i j}=h_{\tau} X\left(A_{i}+\alpha I\right) \text {, }
$$$$
\Pi_{22}^{4 i j}
$$$$
=-2 \bar{R}+\bar{U}+\bar{U}^{T}-\bar{V}_{2}-\bar{V}_{2}^{T}-h_{\tau} \bar{T}-\frac{\pi^{2}}{4} W
$$$$
+\sigma \exp (2 \alpha \tau(t)) \bar{\Phi}
$$$$
\Pi_{23}^{4 i j}=\bar{R}-\bar{U}-\bar{V}_{3}^{T} \text {, }
$$$$
\Pi_{25}^{4 i j}=h_{\tau} \exp (\alpha \tau(t))\left\{\Upsilon_{q} \bar{K}_{j}+\Upsilon_{q}^{-} \bar{F}_{j}\right\}^{T} L^{T} B_{i}^{T},
$$$$
\Pi_{33}^{4 i j}=-\bar{Q}-\bar{R}
$$$$
\Pi_{44}^{4 i j}=-\bar{\Phi},
$$$$
\Pi_{55}^{4 i j}=\bar{R}+\bar{W}-2 X
$$$$
\bar{V}=\left[\begin{array}{lllll}
\bar{V}_{1} & \bar{V}_{2} & \bar{V}_{3} & 0 & 0
\end{array}\right]^{T},
$$

$$
\begin{aligned}
& \Lambda_{5}^{i j}=\left[X\left(A_{i}+\alpha I\right)^{T} \quad \lambda_{1} \quad 0 \quad 000\right], \\
& \Gamma_{0}^{i j}=\left[\begin{array}{ccccc}
M^{T} & 0 & 0 & 0 & h_{\tau} M^{T} \\
E_{a i} X & \lambda_{2} & 0 & 0 & 0
\end{array}\right]^{T} \text {, } \\
& \widehat{\Gamma}_{0}^{i j}=\left[\begin{array}{ccccc}
M^{T} & 0 & 0 & h_{\tau} M^{T} & h_{\tau} M^{T} \\
E_{a i} X & \lambda_{2} & 0 & 0 & 0
\end{array}\right]^{T} \text {, } \\
& \Xi_{0}^{i j}=\operatorname{diag}\left\{-\varepsilon_{i j} I-\varepsilon_{i j}^{-1} I\right\} \text {, } \\
& \lambda_{1}=\exp (\alpha \tau(t))\left\{\Upsilon_{q} \bar{K}_{j}+\Upsilon_{q}^{-} \bar{F}_{j}\right\}^{T} L^{T} B_{i}^{T}, \\
& \lambda_{2}=\exp (\alpha \tau(t)) E_{b i} L\left\{\Upsilon_{q} \bar{K}_{j}+\Upsilon_{q}^{-} \bar{F}_{j}\right\}
\end{aligned}
$$

then the nonuniform transmission nonlinear NCS (17) remains asymptotically stable in the domain of attraction $\varepsilon(P)$ and possesses $\alpha$-safety degree. $K$ and $\Phi$ are the group of the fault-tolerant controller and the event-triggered weight matrix. Furthermore, we can obtain the fault-tolerant controller and the event-triggered matrix by $K_{j}=\bar{K}_{j} X^{-1}$ and $\Phi=X^{-1} \bar{\Phi} X^{-1}$, respectively.

Proof. We replace $\bar{A}, \bar{B}$ with $A_{i}+\Delta A_{i}+\alpha I$ and $\exp (\alpha \tau(t))\left(B_{i}+\right.$ $\left.\Delta B_{i}\right) L$ in (24), respectively. Based on (3) and Lemma 9 of [25], we can obtain

$$
\left[\begin{array}{cc}
\Pi^{5 i j} & h_{\tau} \widehat{V} \\
* & -h_{\tau} S
\end{array}\right]+\varepsilon_{i j}^{-1} \Gamma_{1}^{i j} \Gamma_{1}^{i j T}+\varepsilon_{i j} \Psi_{1}^{i j} \Psi_{1}^{i j T}<0,
$$

where

$$
\begin{aligned}
\Pi_{11}^{5 i j} & \\
= & P\left(A_{i}+\alpha I\right)+\left(A_{i}+\alpha I\right)^{T} P+Q-\frac{\pi^{2}}{4} W-R \\
& +V_{1}+V_{1}^{T}, \\
\Pi_{12}^{5 i j} & \\
= & \exp (\alpha \tau(t)) P B_{i} L\left\{\Upsilon_{q} K_{j}+\Upsilon_{q}^{-} F_{j}\right\}+\frac{\pi^{2}}{4} W+R \\
& -U-V_{1}+V_{2}^{T}, \\
\Pi_{13}^{5 i j}= & U+V_{3}^{T}, \\
\Pi_{14}^{5 i j}= & h_{\tau}\left(A_{i}+\alpha I\right)^{T}, \\
\Pi_{22}^{5 i j}= & -h_{\tau} T-\frac{\pi^{2}}{4} W-2 R+U+U^{T}-V_{2}-V_{2}^{T}, \\
\Pi_{23}^{5 i j}= & R-U-V_{3}^{T}, \\
\Pi_{24}^{5 i j}= & \exp (\alpha \tau(t)) h_{\tau}\left\{\Upsilon_{q} K_{j}+\Upsilon_{q}^{-} F_{j}\right\}^{T} L^{T} B_{i}^{T}, \\
\Pi_{33}^{5 i j}= & -Q-R_{1}, \\
\Pi_{44}^{5 i j}= & -(R+W)^{-1},
\end{aligned}
$$




$$
\begin{aligned}
& \Gamma_{1}^{i j T} \\
& \quad=\left[\begin{array}{lllll}
E_{a i} & \exp (\alpha \tau(t)) E_{b i} L\left\{\Upsilon_{q} K_{j}+\Upsilon_{q}^{-} F_{j}\right\} & 0 & 0 & 0
\end{array}\right], \\
& \Psi_{1}^{i j T}=\left[\begin{array}{lllll}
M^{T} P & 0 & 0 & h_{\tau} M^{T} & 0
\end{array}\right]
\end{aligned}
$$

$\varepsilon_{i j}$ is some scalar, and $\varepsilon_{i j 9}>0$.

With regard to the Schur Complement Lemma, (59) is equivalent to

$$
\left[\begin{array}{ccc}
\Pi^{6 i j} & h_{\tau} \widehat{V} & \Gamma_{2}^{i j} \\
* & -h_{\tau} S & 0 \\
* & * & \Xi_{1}^{i j}
\end{array}\right]<0,
$$

where

$$
\begin{aligned}
& \Pi^{6 i j}=\Pi^{5 i j}, \\
& \Xi_{1}^{i j}=\Xi_{0}^{i j}, \\
& \Gamma_{2}^{i j} \\
& =\left[\begin{array}{ccccc}
M^{T} P & 0 & 0 & h_{\tau} M^{T} & 0 \\
E_{a i} & \exp (\alpha \tau(t)) E_{b i} L\left\{\Upsilon_{q} K_{j}+\Upsilon_{q}^{-} F_{j}\right\} & 0 & 0 & 0
\end{array}\right]^{T} .
\end{aligned}
$$

Transforming matrix inequality (61) into the corresponding linear matrix inequality through congruent transformation is necessary to conveniently solve matrix inequality. Pre- and postmultiplying $\left[\begin{array}{l}R U \\ * R\end{array}\right] \geq 0$ with $J_{1}=\operatorname{diag}\left\{P^{-1}, P^{-1}\right\}$ result in (54) when we define $\bar{R}=X R X, \bar{U}=X U X$.

Pre- and postmultiplying (61) with $J_{1}=\operatorname{diag}\left\{P^{-1}, P^{-1}\right.$, $\left.P^{-1}, I, P^{-1}, I, I\right\}$, we can obtain

$$
\left[\begin{array}{ccc}
\Pi^{7 i j} & h_{\tau} P^{-1} \widehat{V} P^{-1} & \Gamma_{3}^{i j} \\
* & -h_{\tau} P^{-1} S P^{-1} & 0 \\
* & * & \Xi_{1}^{i j}
\end{array}\right]<0,
$$

where

$$
\begin{aligned}
\Pi_{11}^{7 i j}= & \left(A_{i}+\alpha I\right) P^{-1}+P^{-1}\left(A_{i}+\alpha I\right)^{T}+P^{-1} Q P^{-1} \\
& -\frac{\pi^{2}}{4} P^{-1} W P^{-1}-P^{-1} R P^{-1}+P^{-1} V_{1} P^{-1}, \\
\Pi_{12}^{7 i j}= & \exp (\alpha \tau(t)) B_{i} L\left\{\Upsilon_{q} K_{j} P^{-1}+\Upsilon_{q}^{-} F_{j} P^{-1}\right\} \\
& +\frac{\pi^{2}}{4} P^{-1} W P^{-1}+P^{-1} R P^{-1}-P^{-1} U P^{-1} \\
& -P^{-1} V_{1} P^{-1}+P^{-1} V_{2}^{T} P^{-1}, \\
\Pi_{13}^{7 i j}= & P^{-1} U P^{-1}+P^{-1} V_{3}^{T} P^{-1}, \\
\Pi_{14}^{7 i j}= & h_{\tau} P^{-1}\left(A_{i}+\alpha I\right)^{T},
\end{aligned}
$$

$$
\begin{aligned}
& \Pi_{22}^{7 i j}=-h_{\tau} P^{-1} T P^{-1}-\frac{\pi^{2}}{4} P^{-1} W P^{-1}-2 P^{-1} R P^{-1} \\
& +P^{-1} U P^{-1}-P^{-1} U^{T} P^{-1}-P^{-1} V_{1} P^{-1} \\
& -P^{-1} V_{2}^{T} P^{-1}, \\
& \Pi_{23}^{7 i j}=P^{-1} R P^{-1}-P^{-1} U P^{-1}-P^{-1} V_{3}^{T} P^{-1} \text {, } \\
& \Pi_{24}^{7 i j}=\exp (\alpha \tau(t)) h_{\tau} P^{-1}\left\{\Upsilon_{q} K_{j}+\Upsilon_{q}^{-} F_{j}\right\}^{T} L^{T} B_{i}^{T}, \\
& \Pi_{33}^{7 i j}=-P^{-1} Q P^{-1}-P^{-1} R P^{-1} \text {, } \\
& \Pi_{44}^{7 i j}=-(R+W)^{-1}, \\
& \Gamma_{3}^{i j}=\left[\begin{array}{ccccc}
M^{T} & 0 & 0 & h_{\tau} M^{T} & 0 \\
E_{a i} P^{-1} & \lambda_{3} & 0 & 0 & 0
\end{array}\right]^{T}, \\
& \lambda_{3}=\exp (\alpha \tau(t)) E_{b i} L\left\{\Upsilon_{q} K_{j}+\Upsilon_{q}^{-} F_{j}\right\} P^{-1} .
\end{aligned}
$$

Define $P^{-1}=X, \bar{R}=X R X, \bar{Q}=X Q X, \bar{S}=X S X, \bar{T}=$ $X T X, \bar{W}=X W X, \bar{K}_{j}=K_{j} X, \bar{F}_{j}=F_{j} X(j=1,2, \ldots, r)$, $\bar{V}=X \widehat{V} X$, and $\bar{\Phi}=X \Phi X$.

For the matrix $N$ with appropriate dimension, if $\left(N^{-1}-\right.$ $\left.P^{-1}\right) N\left(N^{-1}-P^{-1}\right) \geq 0$ for $N^{-1}>0$, then $-P^{-1} N P^{-1} \leq N^{-1}-$ $2 P^{-1}$. We obtain $-(R+W)^{-1} \leq \bar{R}+\bar{W}-2 X,-S^{-1} \leq \bar{S}-2 X$. Therefore, we can obtain (55). Similarly, we can obtain (56) using a series of processing methods.

When the actuator has saturation constraint, we make some mathematical transitions to the linear domain condition.

$$
\varepsilon(P) \subset \ell(F) \Longleftrightarrow\left|f_{l} X\right| \leq 1, \quad \forall x \in \varepsilon(P),
$$

where $f_{l}$ is the $l$ th row of the matrix $F$ for $l \in[1, m]$. Equation (65) is equivalent to

$$
f_{l} P^{-1} f_{l}^{T} \leq 1 .
$$

In addition, applying the Schur Complement into (66), we can obtain

$$
\left[\begin{array}{cc}
1 & f_{l} P^{-1} \\
* & P^{-1}
\end{array}\right] \geq 0, \quad l \in[1, m] .
$$

As $P^{-1}=X, \bar{F}_{j}=F_{j} X$ are defined as above, (67) is equivalent to (57). Namely, when the system parameters satisfy (54)-(57), the control law (15) can make the state trajectories of the nonlinear NCS (17) remain inside the ellipsoid $\varepsilon(P)$ and possess a $\alpha$-safety degree. Meanwhile, we can get the nonuniform transmission robust fault-tolerant controller gain matrix and event-triggered weight matrices by $K_{j}=\bar{K}_{j} X^{-1}$ and $\Phi=X^{-1} \bar{\Phi} X^{-1}$. The proof is completed.

3.2.2. The Codesign Method between Fault-Tolerance and Communication for Closed-Loop Failure System (17) with Multiobjective Constraint. The codesign goal of multiobjective 
constraint fault-tolerant control under DETCS is to obtain the appropriate multiobjective constraint fault-tolerant control gain and the event-triggered weight matrix for system (17), thereby making the system asymptotically stable and obtaining some other performance indexes such as $\alpha$-safety degree, $\mathrm{H}_{\infty}$-performance, $\mathrm{H}_{2}$-performance, and fewer occupancy of network communication resource.

Theorem 9. Based on the event-triggered condition (6) of the DETCS, there exist some matrices $X=X^{T}>0, \breve{\Phi}=\breve{\Phi}^{T}>$ $0, \breve{Q}, \breve{R}, \breve{S}, \breve{T}, \breve{W}, \breve{K}_{j}(j=1,2, \ldots, r), \breve{U}$ and $\breve{V}$ for the given values $\sigma, h_{\tau}, \alpha, \gamma_{1}$, and $\gamma_{2}$. If all the given values and the matrix variables satisfy $\varepsilon(P) \subset \ell(F)$ and the matrix inequalities

$$
\begin{aligned}
& {\left[\begin{array}{cc}
\breve{R} & \breve{U} \\
* & \breve{R}
\end{array}\right] \geq 0,} \\
& {\left[\begin{array}{ccc}
\Pi^{8 i j} & h_{\tau} \breve{V} & \Gamma_{3}^{i j} \\
* & -h_{\tau} \breve{S} & 0 \\
* & * & \Xi_{2}^{i j}
\end{array}\right]<0,} \\
& {\left[\begin{array}{ccc}
\Pi^{8 i j} & h_{\tau} \Lambda_{6}^{i j} & \Gamma_{3}^{i j} \\
* & h_{\tau} \breve{S}-2 h_{\tau} X & 0 \\
* & * & \Xi_{2}^{i j}
\end{array}\right]<0,} \\
& {\left[\begin{array}{cc}
X & X C_{i 2}^{T} \\
* & \gamma_{2}^{2} I
\end{array}\right]>0 \text {, }} \\
& {\left[\begin{array}{cc}
1 & \breve{f}_{l} \\
* & X
\end{array}\right] \geq 0, \quad \text { for } l \in[1, m]}
\end{aligned}
$$

the nonuniform transmission nonlinear NCS (17) can remain asymptotically stable in the domain of attraction $\varepsilon(P)$ and possesses $\alpha$-safety degree, $\gamma_{1}$-disturbance rejection level, $\gamma_{2}$-outpeak rejection level, and less data transmission occupancy rate. $K$ and $\Phi$ are the group of the fault-tolerant controller and the event-triggered weight matrix. Furthermore, we can obtain the fault-tolerant controller and the event-triggered matrix by $K_{j}=\bar{K}_{j} X^{-1}$ and $\Phi=X^{-1} \bar{\Phi} X^{-1}$, respectively, where

$$
\begin{aligned}
\Pi_{11}^{8 i j}= & \left(A_{i}+\alpha I\right) X+X\left(A_{i}+\alpha I\right)^{T}+\breve{Q}-\breve{R}+\breve{V}_{1} \\
& +\breve{V}_{1}^{T}-\frac{\pi^{2}}{4} \breve{W}, \\
\Pi_{12}^{8 i j}= & \exp (\alpha \tau(t)) B_{i} L\left\{\Upsilon_{q} \breve{K}_{j}+\Upsilon_{q}^{-} \breve{F}_{j}\right\}+\breve{R}-\breve{U}-\breve{V}_{1} \\
& +\breve{V}_{2}^{T}+\frac{\pi^{2}}{4} \breve{W}, \\
\Pi_{13}^{8 i j}= & \breve{U}+\breve{V}_{3}^{T}, \\
\Pi_{15}^{8 i j}= & B_{w i}, \\
\Pi_{16}^{8 i j}= & X C_{i 1}^{T},
\end{aligned}
$$

$$
\begin{aligned}
& \Pi_{17}^{8 i j}=h_{\tau} X\left(A_{i}+\alpha I\right)^{T}, \\
& \Pi_{22}^{8 i j}=-2 \breve{R}+\breve{U}+\breve{U}^{T}-\breve{V}_{2}-\breve{V}_{2}^{T}+h_{\tau} \breve{T}-\frac{\pi^{2}}{4} \breve{W} \\
& +\sigma \exp (2 \alpha \tau(t)) \breve{\Phi} \\
& \Pi_{23}^{8 i j}=\breve{R}-\breve{U}-\breve{V}_{3}^{T} \text {, } \\
& \Pi_{27}^{8 i j}=h_{\tau} \exp (\alpha \tau(t))\left\{\Upsilon_{q} \breve{K}_{j}+\Upsilon_{q}^{-} \breve{F}_{j}\right\}^{T} B_{i}^{T} L, \\
& \Pi_{33}^{8 i j}=-\breve{Q}-\breve{R}, \\
& \Pi_{44}^{8 i j}=-\breve{\Phi}, \\
& \Pi_{55}^{8 i j}=-\gamma_{1}^{2} I, \\
& \Pi_{57}^{8 i j}=h_{\tau} B_{w i}^{T} \text {, } \\
& \Pi_{66}^{8 i j}=-e_{i} I \text {, } \\
& \Pi_{77}^{8 i j}=\bar{R}+\bar{W}-2 X \text {, } \\
& \breve{V}=\left[\begin{array}{lllllll}
V_{1} & V_{2} & V_{3} & 0 & 0 & 0 & 0
\end{array}\right]^{T},
\end{aligned}
$$

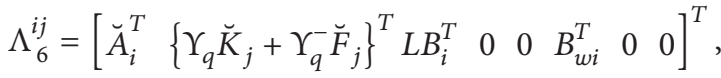

$$
\begin{aligned}
& \Gamma_{3}^{i j}=\left[\begin{array}{ccccccc}
M & 0 & 0 & 0 & 0 & h_{\tau} M & h_{\tau} M \\
X E_{a i}^{T} & \lambda_{3} & 0 & 0 & 0 & 0 & 0
\end{array}\right]^{T} \text {, } \\
& \Xi_{2}^{i j}=\operatorname{diag}\left\{-\varepsilon_{i j} I,-\varepsilon_{i j}^{-1} I\right\} \text {, } \\
& \hbar_{3}=\exp (\alpha \tau(t)) B_{i} L\left\{\Upsilon_{q} \breve{K}_{j}+\Upsilon_{q}^{-} \breve{F}_{j}\right\}^{T} L E_{b i}^{T} .
\end{aligned}
$$

The proof process is similar to Theorem 8 . The proof detail is omitted herein because of the limited space, and the proof process is detailed in Appendix.

Remark 10. There are four theorems. Theorems 6 and 7 are two stability criterions. Suppose that the fault-tolerant controller and the event-triggered weight matrix are previously provided and determine if the closed-loop failure system (17) with nonuniform transmission period satisfies the related performance index simultaneously, such as $\alpha$-safety degree, $\mathrm{H}_{\infty}$-performance, $\mathrm{H}_{2}$-performance, and less data transmission. Furthermore, Theorems 8 and 9 are the related codesign method between control and communication. Particularly, the multiobjective constraint fault-tolerant controller and event-triggered weight matrix are simultaneously obtained by solving the related linear matrix inequalities in Theorems 8 and 9, which can make system (17) possess some desired performance indexes. Therefore, Theorems 6 and 7 are the criterions of judgment and Theorems 8 and 9 are the methods of solving controller and event-triggered weight matrix. From the point of the theorem proof process, the proof of Theorems 6 and 7 are the bases of Theorems 8 and 9 . 
If the parameters $\alpha, \sigma, \gamma_{1}$, and $\gamma_{2}$ are provided in advance, the satisfactory fault-tolerant controller and the event-triggered weight matrix which are solved through
Theorem 9 are only $\gamma_{i}$-suboptimal solutions for system (17) under the DETCS. $\gamma_{j}(i, j=1,2, i \neq j)$ can be optimized by (74) for $\alpha, \sigma$, and $\gamma_{i}$ provided in advance

$$
\begin{array}{cl}
\min _{h_{\tau}, \sigma, \alpha, \gamma_{i}} & \gamma_{j} \\
\text { s.t. } & (69) \sim(73), \quad X>0 \\
& \breve{Q}>0, \breve{R}>0, \breve{S}>0, \breve{T}>0, \breve{W}>0, \breve{U}>0, \breve{V}>0 .
\end{array}
$$

In addition, we can obtain the optimal fault-tolerant controller and event-triggered weight matrix for system (17) with nonuniform transmission period. If some parameters are provided in advance, the corresponding optimal faulttolerant controller and the event-triggered weight matrix under the responding optimal performance by a similar method can be obtained along with other optimal performance indexes.

\section{Simulation Experiment}

Considering the uncertain nonlinear NCS model [32], if we choose $M_{1}\left(x_{2}\right)=\sin ^{2} x_{2}$ and $M_{2}\left(x_{2}\right)=\cos ^{2} x_{2}$ as fuzzy membership function, then the fuzzy system with two rules is expressed as follows.

Rule $i$. If $x_{2}$ is $M_{i}, i=1,2$, then

$$
\begin{aligned}
\dot{x}(t)= & \left(A_{i}+\Delta A_{i}\right) x(t)+\left(B_{i}+\Delta B_{i}\right) \operatorname{sat}(u(t)) \\
& +B_{w i} w(t), \\
z_{1}(t)= & C_{i 1} x(t), \\
z_{2}(t)= & C_{i 2} x(t), \\
A_{1}= & {\left[\begin{array}{cc}
-3 & 1 \\
1 & -1
\end{array}\right], } \\
A_{2}= & {\left[\begin{array}{cc}
-2 & 1 \\
1 & 0
\end{array}\right], } \\
B_{1}= & {\left[\begin{array}{cc}
1 & 0 \\
0 & -0.5
\end{array}\right], } \\
B_{2}= & {\left[\begin{array}{ll}
1 & 0 \\
0 & 0.5
\end{array}\right], } \\
B_{w 1}= & {\left[\begin{array}{c}
1 \\
0.2
\end{array}\right], } \\
B_{w 2}= & {\left[\begin{array}{l}
-1 \\
0.1
\end{array}\right], }
\end{aligned}
$$

$$
\begin{aligned}
C_{11} & =\left[\begin{array}{cc}
1 & 0 \\
0 & -0.5
\end{array}\right], \\
C_{21} & =\left[\begin{array}{cc}
1 & 0 \\
0 & 0.5
\end{array}\right], \\
C_{12} & =\left[\begin{array}{cc}
0.9 & 0 \\
0 & -0.5
\end{array}\right], \\
C_{22} & =\left[\begin{array}{cc}
0.9 & 0 \\
0 & 0.8
\end{array}\right], \\
w(t) & =\left\{\begin{array}{cc}
\sin (2 \pi t) \exp (-0.2 t), & 10 \leq t \leq 20 \\
0 & \text { other. }
\end{array}\right.
\end{aligned}
$$

The uncertain parameter matrix $\Delta A_{i}, \Delta B_{i}(i=1,2)$ satisfy condition (3), and the related parameters in (3) are

$$
\begin{aligned}
M & =\left[\begin{array}{cc}
0.31 & 0.1 \\
0 & 0
\end{array}\right], \\
F(t) & =\left[\begin{array}{cc}
\sin t & 0 \\
0 & \cos t
\end{array}\right], \\
E_{a i} & =\left[\begin{array}{cc}
0 & 0.2 \\
0 & 0
\end{array}\right], \\
E_{b i} & =\left[\begin{array}{cc}
0 & 0.2 \\
0 & 0
\end{array}\right] .
\end{aligned}
$$

The eigenvalues of system matrices $A_{1}$ and $A_{2}$ are -3.4142 , $-0.5858,-2.4142$, and 0.4142 . The system is unstable if the control input is not placed in the system. The values of actuator failure matrix are $L_{0}=\operatorname{diag}\{1,1\}, L_{1}=\operatorname{diag}\{0,1\}$, $L_{2}=\operatorname{diag}\{0.8,0.5\}$, and $L_{3}=\operatorname{diag}\{0.4,0.9\}$. We select $x_{0}=$ $[1,-1]^{T}, \hbar=0.1, h_{\max k}=0.6, \tau(t)=0.07+0.03|\sin t|$, $\bar{\tau}=0.1 \mathrm{~s}, h_{\tau}=h_{\max k}+\bar{\tau}=0.7 \mathrm{~s}$, and $\sigma=0.05$. The simulation experiment was conducted in three cases to completely verify the effectiveness of the obtained theory result. Case 1 is the circumstance of system (17) only with $\alpha$-safety degree. Case 2 is the circumstance of system (17) with $\alpha$-safety degree and $H_{\infty}$ performance index. Case 3 is the circumstance of system 
(17) with $\alpha$-safety degree, $H_{\infty}$ performance index, and $H_{2}$ performance index.

Case 1. Based on a discrete event-triggered condition (6), if $\alpha=0.1$, we can obtain the fault-tolerant control gain matrix $K_{11}, K_{21}$, and the event-triggered weight matrix $\Phi_{1}$ by Theorem 8 .

$$
\begin{aligned}
& K_{11}=\left[\begin{array}{cc}
0.0327 & -0.3774 \\
-0.5578 & 0.0906
\end{array}\right], \\
& K_{21}=\left[\begin{array}{cc}
0.0514 & -0.3217 \\
-0.4697 & 0.0763
\end{array}\right], \\
& \Phi_{1}=\left[\begin{array}{cc}
0.9631 & -0.2581 \\
-0.2581 & 0.4761
\end{array}\right] .
\end{aligned}
$$

Case 2. Based on the discrete event-triggered condition (6), if $\alpha=0.1$ and $\gamma_{1}=1.3$, we can obtain the fault-tolerant control gain matrix $K_{12}, K_{22}$, and the event-triggered weight matrix $\Phi_{2}$ by Theorem 9 (without considering (69)).

$$
\begin{aligned}
K_{12} & =\left[\begin{array}{ll}
-0.5753 & -1.9147 \\
-1.0985 & -0.8791
\end{array}\right], \\
K_{22} & =\left[\begin{array}{ll}
-0.5758 & -1.9097 \\
-1.0944 & -0.8758
\end{array}\right], \\
\Phi_{2} & =\left[\begin{array}{ll}
2.3374 & -1.3976 \\
-1.3976 & 1.2947
\end{array}\right] .
\end{aligned}
$$

Case 3. Based on the discrete event-triggered condition (6), if $\alpha=0.1, \gamma_{1}=1.3$, and, $\gamma_{2}=1.3$, we can obtain the faulttolerant control gain matrix $K_{13}, K_{23}$, and the event-triggered weight matrix $\Phi_{3}$ by Theorem 9 .

$$
\begin{aligned}
K_{13} & =\left[\begin{array}{ll}
-0.5437 & -1.8642 \\
-1.1723 & -0.7733
\end{array}\right], \\
K_{23} & =\left[\begin{array}{ll}
-0.5451 & -1.8623 \\
-1.1701 & -0.7719
\end{array}\right], \\
\Phi_{3} & =\left[\begin{array}{cc}
2.4196 & -1.4267 \\
-1.4267 & 1.1817
\end{array}\right] .
\end{aligned}
$$

There are system state response and control input variety for the three preceding cases which are shown in Figures 3, 4, and 5 .

The codesign method presented in this study is between fault-tolerant control and network communication for the closed-loop failure system (17) with actuator saturation and actuator failure. From Figures 3(a), 4(a), and 5(a), the codesign method under nonuniform transmission can guarantee that the closed-loop failure system (17) is asymptotically stable with some related performance indexes in the three cases. The related performance indexes include $\alpha$-safety degree, disturbance rejection performance, and out-peak rejection
TABLE 1: The system data transmission circumstance in three cases.

\begin{tabular}{lccccc}
\hline & $h_{\tau}$ & $n$ & $\bar{h}$ & $h_{\max }$ & $r_{e / t}$ \\
\hline Case 1 & 0.7 & 156 & 0.1721 & 0.4000 & $52 \%$ \\
Case 2 & 0.7 & 219 & 0.1397 & 0.5000 & $73 \%$ \\
Case 3 & 0.7 & 229 & 0.1367 & 0.7000 & $76.3 \%$ \\
\hline
\end{tabular}

$h_{\tau}$ is the upper bound of $\tau(t) . n$ is the quantity of data transmission; $r_{e / t}$ is the ratio between the quantity of data transmission in the DETCS and the corresponding quantity in the PTTCS; $\bar{h}$ is the average release period; $h_{\max }$ is the maximum release period.

performance. Simultaneously, from Figures 3(b), 4(b), and 5(b), although the system has actuator saturation constraint, the system performance does not deteriorate.

Under the discrete event-triggered condition (6), there are corresponding instant distribution of system data transmission and data transmission interval for the three cases, as shown in Figure 6.

Comparing with PTTCS (300 data transmission), the quantity of data transmission has been significantly reduced in the closed-loop failure system. Based on DETCS, the faulttolerant control for nonuniform transmission nonlinear NCS (17) can save some network communication resource. In addition, the data quantity for transmission becomes substantial with the increase in performance index constraints. Furthermore, the data transmission circumstances of the three cases are shown in Table 1.

Table 1 shows that, with the increase in performance index constraints, the data transmission quantity $n$, the average transmission period $\bar{h}$, and the maximum transmission period $h_{\text {max }}$ become substantial while the saving ratio of data transmission $\left(1-r_{e / t}\right)$ becomes small.

With regard to (74), the optimal $\gamma_{1}$ or $\gamma_{2}$ for the multiobjective constraint fault-tolerant control of Case 3 can be obtained. If $\gamma_{1}=1.3$, the optimal $\gamma_{2 \text { min }}=0.9222$ and the corresponding optimal multiobjective constraint faulttolerant controller $K_{\mathrm{opt} 11}, K_{\mathrm{opt} 21}$, and the event-triggered weight matrix $\Phi_{\text {opt1 }}$ can be obtained

$$
\begin{aligned}
& K_{\text {opt11 }}=\left[\begin{array}{ll}
-0.6372 & -2.3240 \\
-1.4235 & -1.1725
\end{array}\right], \\
& K_{\text {opt21 }}=\left[\begin{array}{ll}
-0.6373 & -2.3237 \\
-1.4232 & -1.1722
\end{array}\right], \\
& \Phi_{\text {opt } 1}=\left[\begin{array}{ll}
0.0909 & -0.0344 \\
-0.0344 & 0.0751
\end{array}\right] .
\end{aligned}
$$

If $\gamma_{2}=1.3$, the optimal $\gamma_{1 \text { min }}=1.1554$ and the corresponding optimal fault-tolerant controller $K_{\text {opt12 }}, K_{\text {opt22 }}$, and the event-triggered weight matrix $\Phi_{\text {opt2 }}$ can be obtained.

$$
\begin{aligned}
& K_{\mathrm{opt} 12}=\left[\begin{array}{ll}
-0.6114 & -1.8763 \\
-1.2709 & -0.9540
\end{array}\right], \\
& K_{\mathrm{opt} 22}=\left[\begin{array}{ll}
-0.6145 & -1.8722 \\
-1.2652 & -0.9497
\end{array}\right],
\end{aligned}
$$




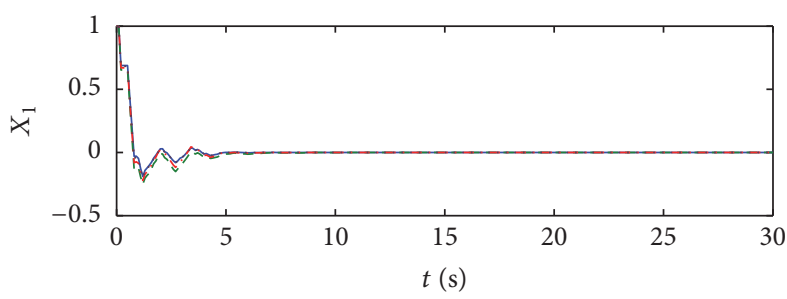

_ Actuator normal $L_{0} \quad \ldots$ Actuator 1, 2 partial failure $L_{2}$ - - - Actuator 1 failure $L_{1} \quad \ldots$... Actuator 1, 2 partial failure $L_{3}$

(a)

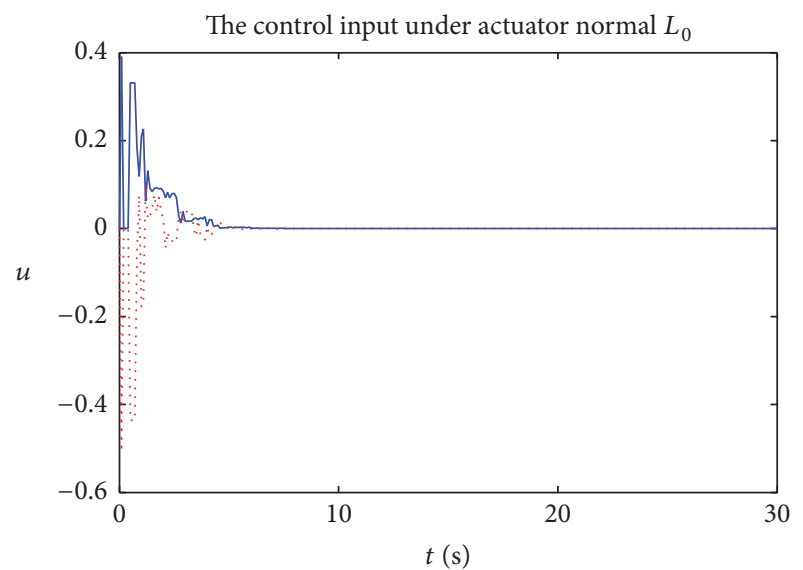

$$
-u_{1}
$$

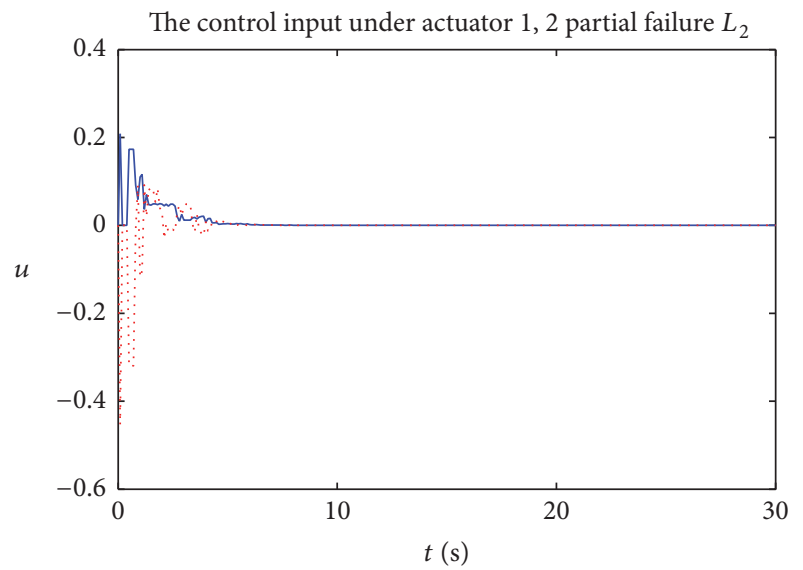

$$
\begin{aligned}
& -u_{1} \\
& \text {.. } u_{2}
\end{aligned}
$$

Figure 3: (a) The state response of the system in Case 1.

$$
\Phi_{\mathrm{opt} 2}=\left[\begin{array}{cc}
0.9209 & -0.5980 \\
-0.5980 & 0.4789
\end{array}\right] .
$$

In Case 3, the correlation for $\alpha$-safety degree and $\gamma_{1 \text { min }}$-optimal disturbance rejection level, $\alpha$-safety degree and $\gamma_{2 \text { min }}$-optimal out-peak rejection level, $\gamma_{1}$-disturbance rejection level and $\gamma_{2 \min }$-optimal out-peak rejection level,

(b)
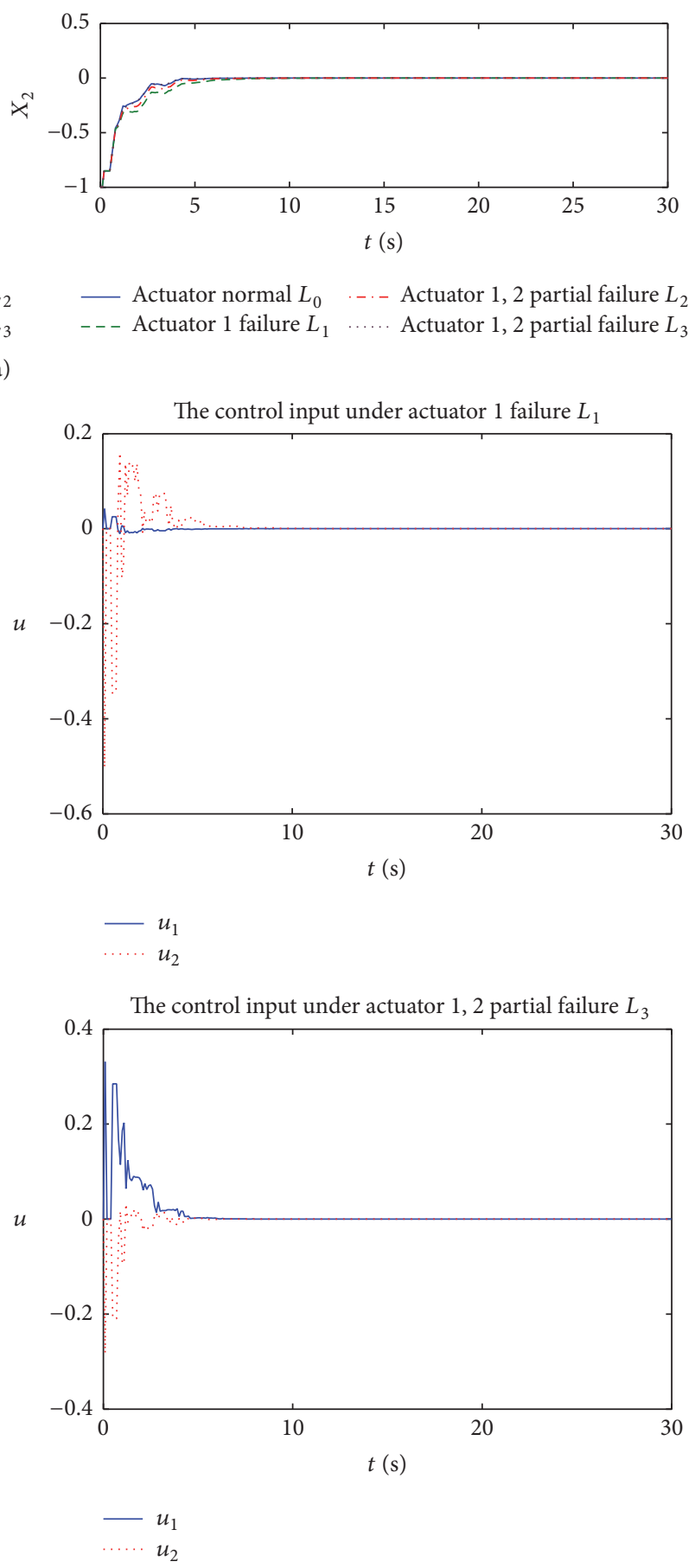

(b) The control input under the actuator saturation in Case 1.

$\gamma_{2}$-out-peak rejection level, and $\gamma_{1 \text { min }}$-optimal disturbance rejection level can be obtained. The related result of the simulation experiment is shown in Table 2.

Table 2 shows the correlation between $\alpha$ and $\gamma_{i \min }(i=$ 1,2). $\gamma_{i}$ and $\gamma_{j \min }(i \neq j, i, j=1,2)$ also have a mutually restricted relationship. Namely, a few compromise balances principles should be regarded in the design process. Sacrificing one kind of performance because of the excessive pursuit 

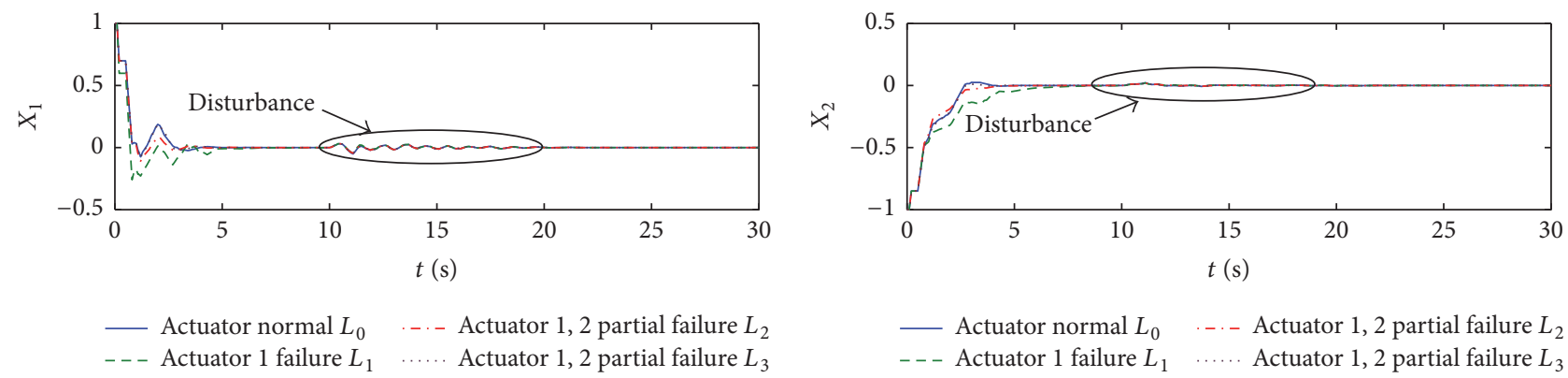

(a)
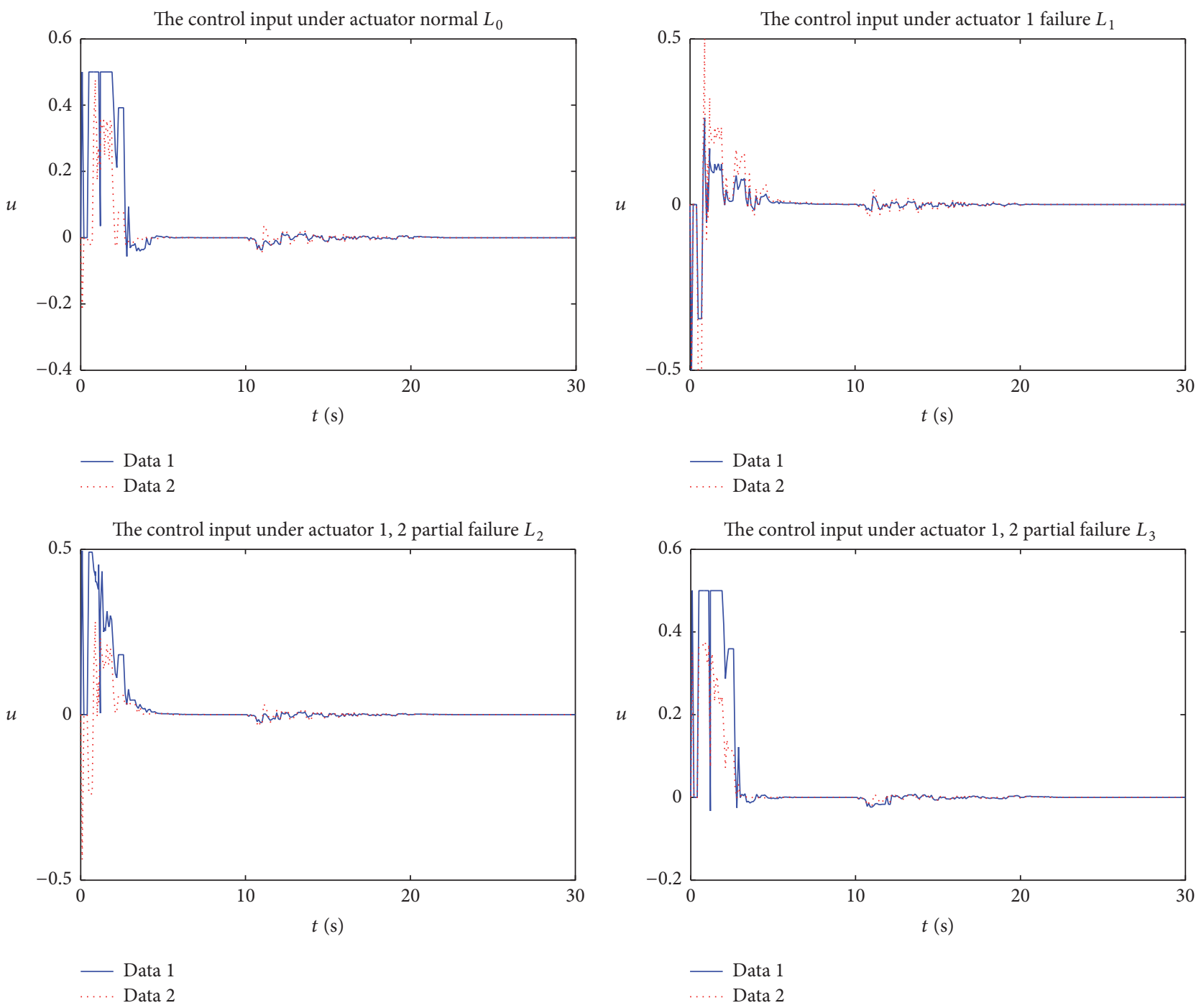

(b)

Figure 4: (a) The state response of the system in Case 2. (b) The control input under the actuator saturation in Case 2.

of the other performance is not necessary. In addition, the distribution circumstance of the feasible solution is obtained, as shown in Figure 7.

Comparing with $[25,30]$, the codesign method provided in the paper under nonuniform transmission can ensure that the closed-loop failure system has a larger maximum allowable time-delay by adopting a discontinuous Lyapunov function (Table 3).

\section{Conclusions}

Considering the circumstance of nonuniform data transmission under DETCS, the codesign problem between multiobjective constraint fault-tolerant control and network communication is investigated for closed-loop failure system with actuator saturation. By doing the comparative analysis between the nonuniform transmission control system and 


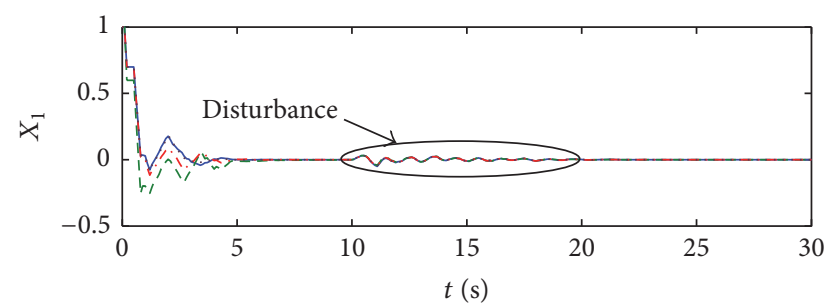

_ Actuator normal $L_{0} \ldots$. - Actuator 1, 2 partial failure $L_{2}$ - - - Actuator 1 failure $L_{1} \quad \ldots$... Actuator 1,2 partial failure $L_{3}$

(a)
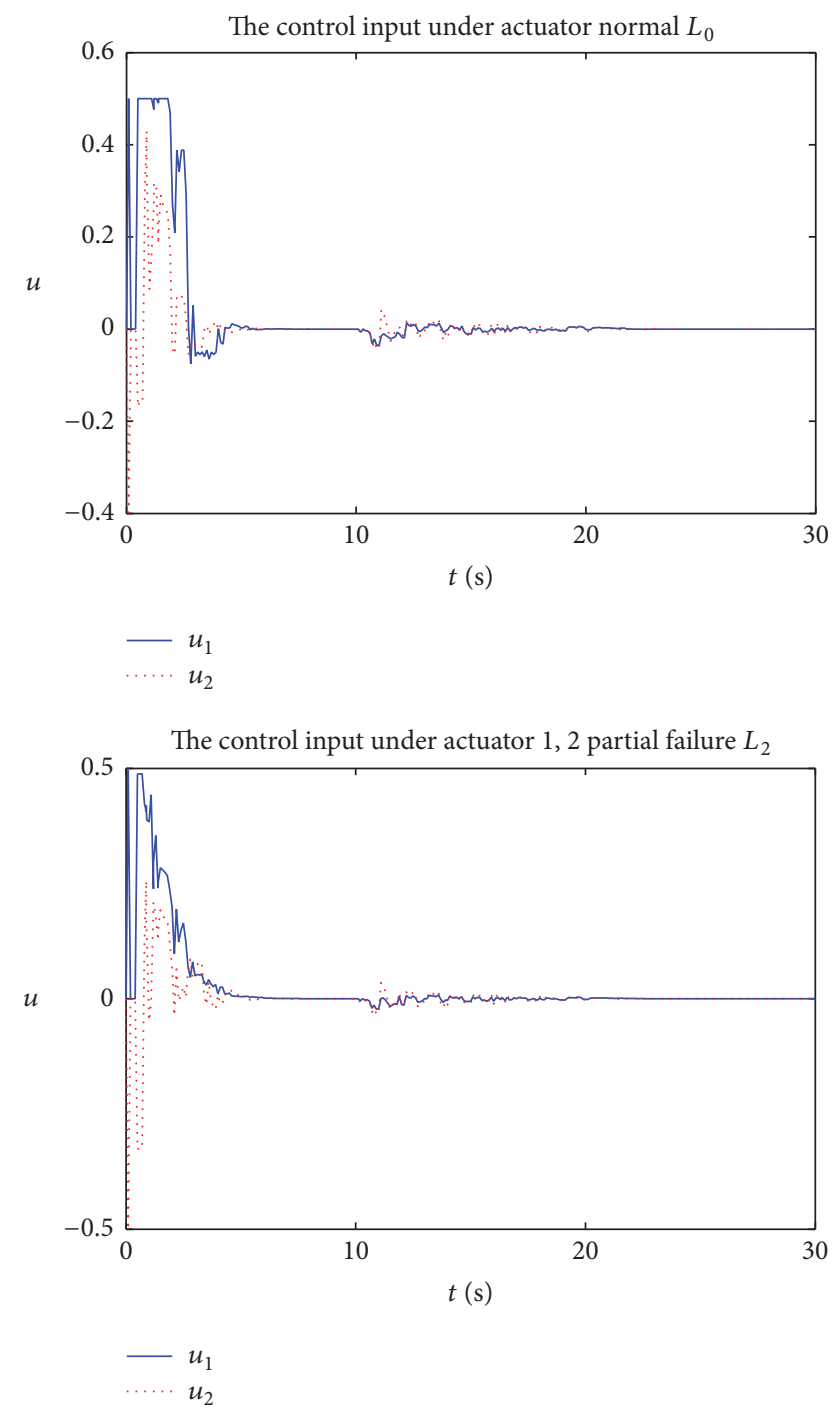

(b)
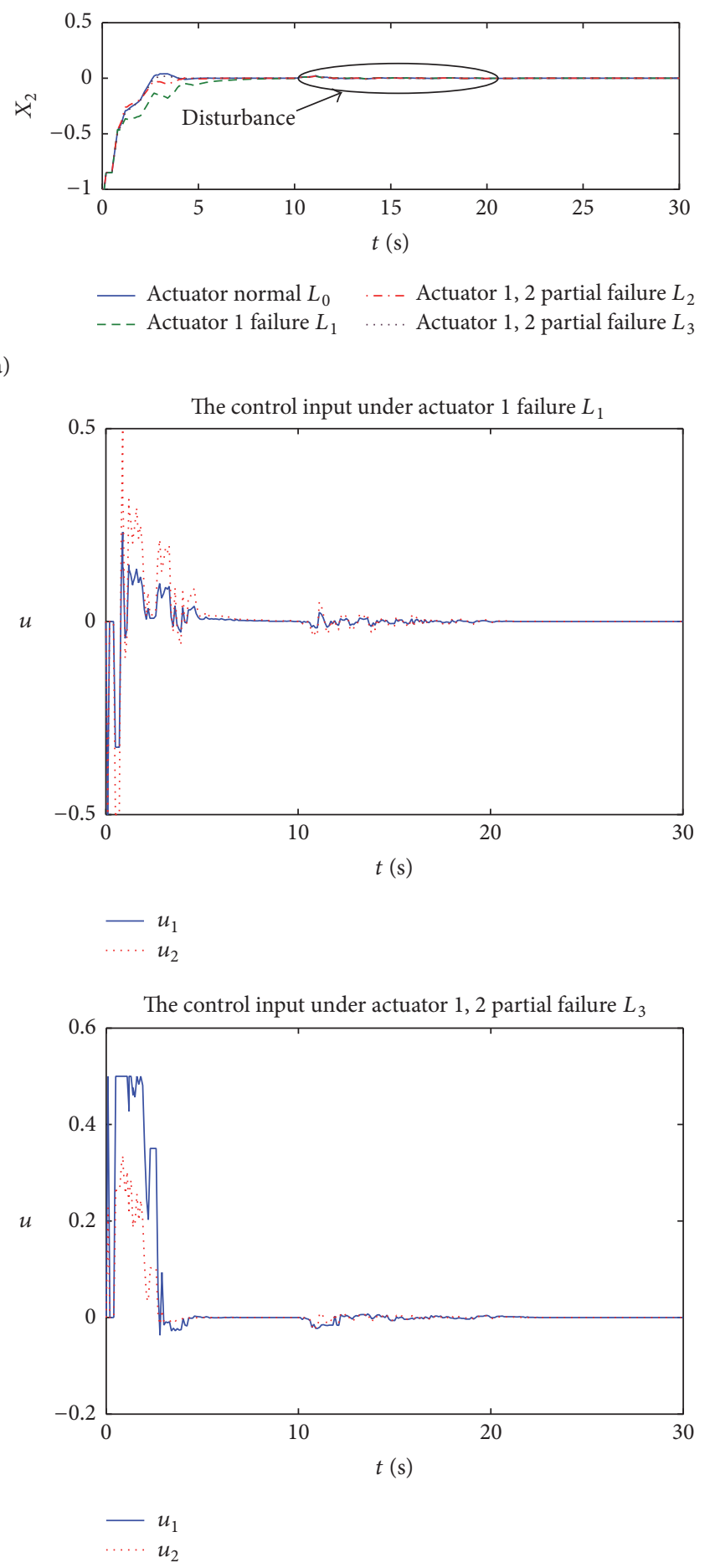

Figure 5: (a) The state response of the system in Case 3. (b) The control input under the actuator saturation in Case 3.

the nonuniform sample-data control system, we can conclude that a similar uncertainty of data updating period at the holder end for these two classes systems exists. The establishment of the closed-loop failure system model was completed based on the related method of sample-data control system. Based on a discontinuous Lyapunov function and the mixed convex combination theory, the stability criterion and the related codesign method are provided by matrix inequalities. The simulation in which the closed-loop failure system satisfies different performance indexes is conducted for the three cases. The simulation result shows that the codesign method does not only guarantee the closed-loop failure 
TABLE 2: The relation among $\alpha, \gamma_{1}, \gamma_{2}, \gamma_{1 \text { min }}$, and $\gamma_{2 \min }$.

\begin{tabular}{lcccc}
\hline$\alpha$ (given) & 0 & 0.1 & 0.2 & - \\
\hline$\gamma_{1 \text { min }}$ & 0.5468 & 1.1554 & 2.6041 & - \\
$\alpha$ (given) & 0 & 0.1 & 0.2 & - \\
$\gamma_{2 \text { min }}$ & 0.7033 & 0.9222 & 1.4760 & - \\
$\gamma_{1}$ (given) & 0.9 & 1.0 & 1.1 & 1.2 \\
$\gamma_{2 \text { min }}$ & 1.4522 & 1.2325 & 1.1028 & 1.0028 \\
$\gamma_{2}$ (given) & 0.7 & 0.8 & 0.9 & 1.0 \\
$\gamma_{1 \text { min }}$ & 2.1127 & 1.8060 & 1.5930 & 1.1359 \\
\hline
\end{tabular}

TABLE 3: The comparison of allowable time-delay under different methods.

\begin{tabular}{lccc}
\hline & $\begin{array}{c}\text { Method } \\
\text { presented in } \\
\text { the paper }\end{array}$ & Reference [30] & Reference [25] \\
\hline $\begin{array}{l}\text { The } \\
\text { maximum } \\
\text { value of } \\
\text { time-delay }\end{array}$ & 0.7 & 0.25 & 0.2 \\
\hline
\end{tabular}

system with some related performance indexes but also saves certain network transmission communication resource. In addition, the codesign method can enable the system with a larger maximum allowable time-delay comparing with the existing results. The research content of the paper belongs to the field of passive fault-tolerant control, whereas the active fault-tolerant control problem will be studied for closedloop failure system in the next step under the nonuniform transmission period of DETCS.

\section{Appendix}

\section{Proof of Theorem 9}

Proof. We replace $\bar{A}, \bar{B}$ with $A_{i}+\Delta A_{i}+\alpha I$ and $\exp (\alpha \tau(t))\left(B_{i}+\right.$ $\left.\Delta B_{i}\right) L$ in (41), respectively. Based on (3) and Lemma 9 of [25], we can obtain

$$
\left[\begin{array}{cc}
\Pi^{9 i j} & h_{\tau} \widehat{V} \\
* & -h_{\tau} S
\end{array}\right]+\varepsilon_{i j 9}^{-1} \Gamma_{9}^{i j} \Gamma_{9}^{i j T}+\varepsilon_{i j 9} \Psi_{9}^{i j} \Psi_{9}^{i j T}<0,
$$

where

$$
\begin{aligned}
& \Pi_{11}^{9 i j}=P\left(A_{i}+\alpha I\right)+\left(A_{i}+\alpha I\right)^{T} P+Q-\frac{\pi^{2}}{4} W-R \\
& \quad+V_{1}+V_{1}^{T}, \\
& \Pi_{12}^{9 i j}=\exp (\alpha \tau(t)) P B_{i} L\left\{\Upsilon_{q} K_{j}+\Upsilon_{q}^{-} F_{j}\right\}+\frac{\pi^{2}}{4} W+R \\
& \quad-U-V_{1}+V_{2}^{T},
\end{aligned}
$$
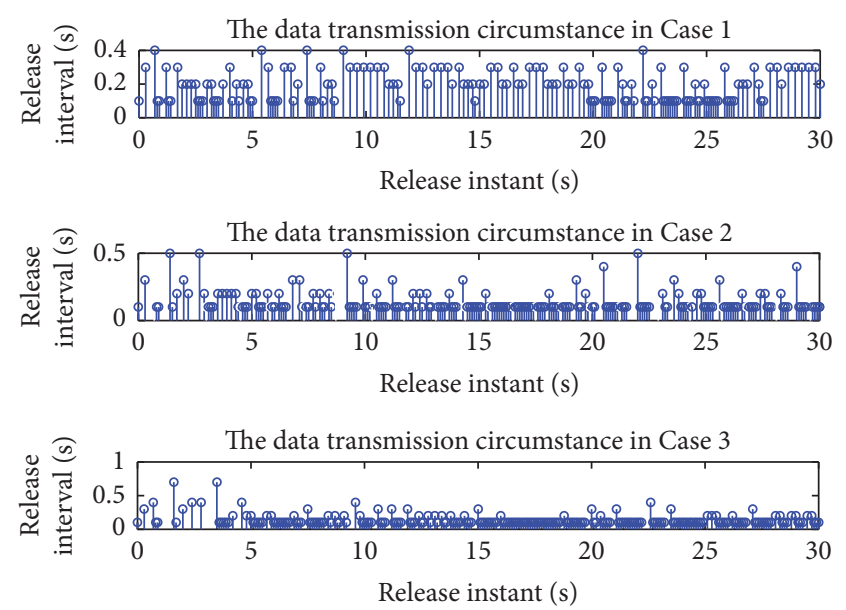

FIGURE 6: The data transmission circumstance of the three cases.

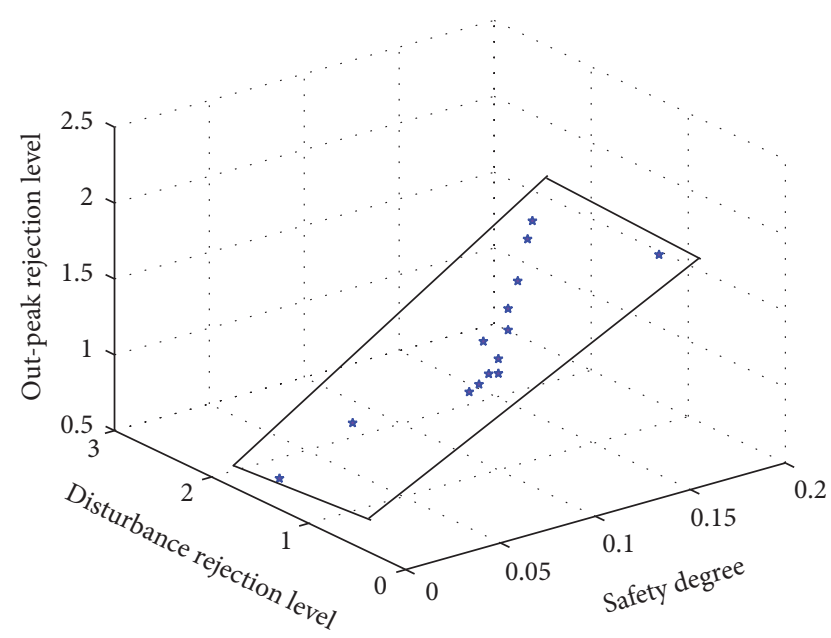

FIGURE 7: The area distribution of the feasible solution in Case 3.

$$
\begin{aligned}
\Pi_{13}^{9 i j} & =U+V_{3}^{T}, \\
\Pi_{15}^{9 i j} & =P B_{w i}, \\
\Pi_{16}^{9 i j} & =C_{i 1}^{T}, \\
\Pi_{17}^{9 i j} & =h_{\tau}\left(A_{i}+\alpha I\right)^{T}, \\
\Pi_{22}^{9 i j} & =-h_{\tau} T-\frac{\pi^{2}}{4} W-2 R+U+U^{T}-V_{2}-V_{2}^{T} \\
& +\sigma \exp (\alpha \tau(t)), \\
\Pi_{23}^{9 i j} & =R-U-V_{3}^{T}, \\
\Pi_{27}^{9 i j} & =\exp (\alpha \tau(t)) h_{\tau}\left\{\Upsilon_{q} K_{j}+\Upsilon_{q}^{-} F_{j}\right\}^{T} B_{i}^{T} L^{T}, \\
\Pi_{33}^{9 i j} & =-Q-R, \\
\Pi_{44}^{9 i j} & =-\Phi, \\
\Pi_{55}^{9 i j} & =-\gamma_{1}^{2} I,
\end{aligned}
$$




$$
\begin{aligned}
& \Pi_{57}^{9 i j}=h_{\tau} B_{w i}^{T}, \\
& \Pi_{66}^{9 i j}=-e_{i} I, \\
& \Pi_{77}^{9 i j}=-(R+W)^{-1}, \\
& \widehat{V}=\left[\begin{array}{lllllll}
V_{1} & V_{2} & V_{3} & 0 & 0 & 0 & 0
\end{array}\right]^{T}, \\
& \Gamma_{9}^{i j T}=\left[\begin{array}{llllllll}
E_{a i} & \lambda_{9} & 0 & 0 & 0 & 0 & 0 & 0
\end{array}\right], \\
& \Psi_{9}^{i j T}=\left[\begin{array}{llllllll}
M^{T} P & 0 & 0 & 0 & 0 & 0 & h_{\tau} M^{T} & 0
\end{array}\right], \\
& \lambda_{9}=\exp (\alpha \tau(t)) E_{b i} L\left\{\Upsilon_{q} K_{j}+\Upsilon_{q}^{-} F_{j}\right\} \text {, } \\
& \varepsilon_{i j 9} \text { is a scalar, } \varepsilon_{i j 9}>0 \text {. }
\end{aligned}
$$

With regard to the Schur Complement Lemma, (A.1) is equivalent to

$$
\left[\begin{array}{ccc}
\Pi^{10 i j} & h_{\tau} \widehat{V} & \Gamma_{10}^{i j} \\
* & -h_{\tau} S & 0 \\
* & * & \Xi_{10}^{i j}
\end{array}\right]<0
$$

where

$$
\begin{aligned}
\Pi^{10 i j} & =\Pi^{9 i j}, \\
\Xi_{10}^{i j} & =\Xi_{0}^{i j}, \\
\Gamma_{10}^{i j} & =\left[\begin{array}{ccccccc}
M^{T} P & 0 & 0 & 0 & 0 & h_{\tau} M^{T} & 0 \\
E_{a i} & \lambda_{10} & 0 & 0 & 0 & 0 & 0
\end{array}\right]^{T}, \\
\lambda_{10} & =\exp (\alpha \tau(t)) E_{b i} L\left\{\Upsilon_{q} K_{j}+\Upsilon_{q}^{-} F_{j}\right\} .
\end{aligned}
$$

Transforming the matrix inequality (A.3) into the corresponding linear matrix inequality through congruent transformation is necessary to conveniently solve matrix inequality (A.3). Pre- and postmultiplying $\left[\begin{array}{ll}R & U \\ * & R\end{array}\right] \geq 0$ with $J_{1}=$ $\operatorname{diag}\left\{P^{-1}, P^{-1}\right\}$, we obtain (68) when we define $\breve{R}=X R X$, $\breve{U}=X U X$.

Pre- and postmultiplying (A.3) with $J_{1}=$ $\operatorname{diag}\left\{P^{-1}, P^{-1}, P^{-1}, P^{-1}, I, I, I, P^{-1}, I, I\right\}$, we obtain

$$
\left[\begin{array}{ccc}
\Pi^{11 i j} & h_{\tau} P^{-1} \widehat{V} P^{-1} & \Gamma_{11}^{i j} \\
* & -h_{\tau} P^{-1} S P^{-1} & 0 \\
* & * & \Xi_{11}^{i j}
\end{array}\right]<0
$$

where

$$
\begin{aligned}
\Pi_{11}^{11 i j}= & \left(A_{i}+\alpha I\right) P^{-1}+P^{-1}\left(A_{i}+\alpha I\right)^{T}+P^{-1} Q P^{-1} \\
& -\frac{\pi^{2}}{4} P^{-1} W P^{-1}-P^{-1} R P^{-1}+P^{-1} V_{1} P^{-1} \\
& +P^{-1} V_{1}^{T} P^{-1}
\end{aligned}
$$

$$
\begin{aligned}
& \Pi_{12}^{11 i j}=\exp (\alpha \tau(t)) B_{i} L\left\{\Upsilon_{q} K_{j}+\Upsilon_{q}^{-} F_{j}\right\} P^{-1} \\
& +\frac{\pi^{2}}{4} P^{-1} W P^{-1}+P^{-1} R P^{-1}-P^{-1} U P^{-1} \\
& -P^{-1} V_{1} P^{-1}+P^{-1} V_{2}^{T} P^{-1}, \\
& \Pi_{13}^{11 i j}=P^{-1} U P^{-1}+P^{-1} V_{3}^{T} P^{-1} \text {, } \\
& \Pi_{15}^{11 i j}=B_{w i}, \\
& \Pi_{16}^{11 i j}=P^{-1} C_{i 1}^{T} \text {, } \\
& \Pi_{17}^{11 i j}=h_{\tau} P^{-1}\left(A_{i}+\alpha I\right)^{T}, \\
& \Pi_{22}^{11 i j}=h_{\tau} P^{-1} T P^{-1}-\frac{\pi^{2}}{4} P^{-1} W P^{-1}-2 P^{-1} R P^{-1} \\
& +P^{-1} U P^{-1}+P^{-1} U^{T} P^{-1}-P^{-1} V_{2} P^{-1} \\
& -P^{-1} V_{2}^{T} P^{-1}+\sigma \exp (\alpha \tau(t)) P^{-1} \Phi P^{-1}, \\
& \Pi_{23}^{11 i j}=P^{-1} R P^{-1}-P^{-1} U P^{-1}-P^{-1} V_{3}^{T} P^{-1}, \\
& \Pi_{27}^{11 i j}=\exp (\alpha \tau(t)) h_{\tau} P^{-1}\left\{\Upsilon_{q} K_{j}+\Upsilon_{q}^{-} F_{j}\right\}^{T} B_{i}^{T} L^{T}, \\
& \Pi_{33}^{11 i j}=-P^{-1} Q P^{-1}-P^{-1} R P^{-1}, \\
& \Pi_{44}^{11 i j}=-P^{-1} \Phi P^{-1}, \\
& \Pi_{55}^{11 i j}=-\gamma_{i}^{2} I, \\
& \Pi_{57}^{11 i j}=h_{\tau} B_{w i}^{T}, \\
& \Pi_{66}^{11 i j}=-e_{i} I \text {, } \\
& \Pi_{77}^{11 i j}=-(R+W)^{-1}, \\
& \Gamma_{11}^{i j}=\left[\begin{array}{ccccccc}
M^{T} & 0 & 0 & 0 & 0 & 0 & h_{\tau} M^{T} \\
E_{a i} P^{-1} & \lambda_{11} & 0 & 0 & 0 & 0 & 0
\end{array}\right]^{T}, \\
& \lambda_{11}=\exp (\alpha \tau(t)) E_{b i} L\left\{\Upsilon_{q} K_{j}+\Upsilon_{q}^{-} F_{j}\right\} P^{-1}, \\
& \Xi_{11}^{i j}=\Xi_{10}^{i j} \text {. }
\end{aligned}
$$

Define $P^{-1}=X, \breve{R}=X R X, \breve{Q}=X Q X, \breve{S}=X S X, \breve{T}=$ $X T X, \breve{U}=X U X, \breve{W}=X W X, \breve{K}_{j}=K_{j} X, \breve{F}_{j}=F_{j} X(j=$ $1,2, \ldots, r)$, and $\breve{\Phi}=X \Phi X$.

For the matrix $N$ with the appropriate dimension, if $\left(N^{-1}-P^{-1}\right) N\left(N^{-1}-P^{-1}\right) \geq 0$ for $N^{-1}>0$, then $-P^{-1} N P^{-1} \leq$ $N^{-1}-2 P^{-1}$. We can obtain $-(R+W)^{-1} \leq \bar{R}+\bar{W}-2 X$, $-S^{-1} \leq \bar{S}-2 X$. Therefore, we can obtain (69). Similarly, we can obtain (70) by a series of processing methods.

Pre- and postmultiplying (43) with $J_{1}=\operatorname{diag}\left\{P^{-1}, I\right\}$, we obtain

$$
\left[\begin{array}{cc}
P^{-1} & P^{-1} C_{i 2}^{T} \\
* & \gamma_{2}^{2} I
\end{array}\right]>0
$$


With regard to $P^{-1}=X, \breve{R}=X R X$, and $\breve{U}=X U X$, we can obtain (71).

When the actuator has saturation constraint, we make some mathematical transition to the linear domain condition

$$
\varepsilon(P) \subset \ell(F) \Longleftrightarrow\left|f_{l} X\right| \leq 1, \quad \forall x \in \varepsilon(P),
$$

where $f_{l}$ is the $l$ th row of the matrix $F$ for $l \in[1, m]$. Equation (A.8) is equivalent to

$$
f_{l} P^{-1} f_{l}^{T} \leq 1
$$

In addition, applying the Schur Complement into (A.9), we obtain

$$
\left[\begin{array}{cc}
1 & f_{l} P^{-1} \\
* & P^{-1}
\end{array}\right] \geq 0, \quad l \in[1, m] .
$$

As $P^{-1}=X$ and $\bar{F}_{j}=F_{j} X$ are defined above, (A.10) is equivalent to (72). Namely, when the system parameters satisfy (68)-(72), the control law (15) can make the state trajectories of the nonlinear NCS (17) remain inside the ellipsoid $\varepsilon(P)$ and possess a $\alpha$-safety degree, $H_{2}$-performance index, and $H_{\infty}$-performance index. Meanwhile, the nonuniform transmission multiobjective constraint fault-tolerant controller gain matrix and event-triggered weight matrices are obtained by $K_{j}=\breve{K}_{j} X^{-1}$ and $\Phi=X^{-1} \breve{\Phi} X^{-1}$. The proof is completed.

\section{Competing Interests}

The authors declare that there is no conflict of interests regarding the publication of this paper.

\section{Acknowledgments}

This work was supported and funded by the National Natural Science of China (Grant nos. 61364011 and 61463030) and the Provincial Natural Science Foundation of Gansu in China (Grant no. 1308RJZA148).

\section{References}

[1] F. Yang, H. Zhang, and Y. Wang, "An enhanced input-delay approach to sampled-data stabilization of T-S fuzzy systems via mixed convex combination," Nonlinear Dynamics, vol. 75 , no. 3 , pp. 501-512, 2014.

[2] S. Bououden, M. Chadli, and H. R. Karimi, "An ant colony optimization-based fuzzy predictive control approach for nonlinear processes," Information Sciences, vol. 299, pp. 143-158, 2015.

[3] S. Aouaouda, M. Chadli, M. T. Khadir, and T. Bouarar, "Robust fault tolerant tracking controller design for unknown inputs T-S models with unmeasurable premise variables," Journal of Process Control, vol. 22, no. 5, pp. 861-872, 2012.

[4] S. Aouaouda, M. Chadli, and H.-R. Karimi, "Robust static output-feedback controller design against sensor failure for vehicle dynamics," IET Control Theory \& Applications, vol. 8, no. 9, pp. 728-737, 2014.
[5] S. Aouaouda, M. Chadli, P. Shi, and H. R. Karimi, "Discretetime $H_{-} / H_{\infty}$ sensor fault detection observer design for nonlinear systems with parameter uncertainty," International Journal of Robust and Nonlinear Control, vol. 25, no. 3, pp. 339-361, 2015.

[6] H. G. Zhang, D. D. Yang, and T. Y. Chai, "Guaranteed cost networked control for T-S fuzzy systems with time delays," IEEE Transactions on Systems, Man and Cybernetics, Part C: Applications and Reviews, vol. 37, no. 2, pp. 160-172, 2007.

[7] C. Peng and T. C. Yang, "Communication-delay-distributiondependent networked control for a class of T-S fuzzy systems," IEEE Transactions on Fuzzy Systems, vol. 18, no. 2, pp. 326-335, 2010.

[8] E. G. Tian and D. Yue, "Reliable $H_{\infty}$ filter design for T-S fuzzy model-based networked control systems with random sensor failure," International Journal of Robust and Nonlinear Control, vol. 23, no. 1, pp. 15-32, 2013.

[9] H. J. Wang, P. Shi, and C.-C. Lim, "Decentralized eventtriggering passive control of networked T-S fuzzy systems," IEEE Transactions on Fuzzy Systems, 2015.

[10] S. L. Hu, D. Yue, C. Peng, X. P. Xie, and X. X. Yin, "Eventtriggered controller design of nonlinear discrete-time networked control systems in T-S fuzzy model," Applied Soft Computing, vol. 30, pp. 400-411, 2015.

[11] C. Peng, Q.-L. Han, and D. Yue, "To transmit or not to transmit: a discrete event-triggered communication scheme for networked takagi-sugeno fuzzy systems," IEEE Transactions on Fuzzy Systems, vol. 21, no. 1, pp. 164-170, 2013.

[12] X.-C. Jia, X.-B. Chi, Q.-L. Han, and N.-N. Zheng, "Eventtriggered fuzzy $H_{\infty}$ control for a class of nonlinear networked control systems using the deviation bounds of asynchronous normalized membership functions," Information Sciences, vol. 259, pp. 100-117, 2014.

[13] H.-Q. Xiao, Y. He, M. Wu, and S.-P. Xiao, " $H_{\infty}$ output tracking control for sampled-data networked control systems in T-S fuzzy model," Acta Automatica Sinica, vol. 41, no. 3, pp. 661-668, 2015.

[14] J. Zhang, H. B. Xu, and H. B. Zhang, "Observer-based sampleddata control for a class of continuous nonlinear systems," Acta Automatica Sinica, vol. 36, no. 12, pp. 1780-1787, 2010.

[15] H. Gao and T. Chen, "Stabilization of nonlinear systems under variable sampling: a fuzzy control approach," IEEE Transactions on Fuzzy Systems, vol. 15, no. 5, pp. 972-983, 2007.

[16] Z. G. Wu, P. Shi, H. L. Su, H. Y. Su, and J. Chu, "Networkbased robust passive control for fuzzy systems with randomly occurring uncertainties," IEEE Transactions on Fuzzy Systems, vol. 21, no. 5, pp. 966-971, 2013.

[17] J. Feng and S.-Q. Wang, "Reliable fuzzy control for a class of nonlinear networked control systems with time delay," Acta Automatica Sinica, vol. 38, no. 7, pp. 1091-1099, 2012.

[18] S. X. Ding, P. Zhang, S. Yin, and E. L. Ding, "An integrated design framework of fault-tolerant wireless networked control systems for industrial automatic control applications," IEEE Transactions on Industrial Informatics, vol. 9, no. 1, pp. 462-471, 2013.

[19] M. S. Mahmoud, A. M. Memon, and P. Shi, "Observer-based fault-tolerant control for a class of nonlinear networked control systems," International Journal of Control, vol. 87, no. 8, pp. 1707$1715,2014$.

[20] M.-Y. Zhao, H.-P. Liu, Z.-J. Li, and D.-H. Sun, "Fault tolerant control for networked control systems with packet loss and time delay," International Journal of Automation and Computing, vol. 8, no. 2, pp. 244-253, 2011. 
[21] E. G. Tian, C. Peng, and Z. Gu, "Fault tolerant control for discrete networked control systems with random faults," International Journal of Control, Automation and Systems, vol. 10, no. 2, pp. 444-448, 2012.

[22] H. J. Wang, B. Zhou, C.-C. Lim, R. Q. Lu, and A. K. Xue, " $H_{\infty}$ fault-tolerant control of networked control systems with actuator failures," IET Control Theory \& Applications, vol. 8, no. 12, pp. 1127-1136, 2014.

[23] H. Huang, D.-X. Xie, D.-F. Zhang, and Z.-Q. Wang, “T-S fuzzy model-based robust $H_{\infty}$ fault-tolerant control for networked control systems," Systems Engineering and Electronics, vol. 32, no. 6, pp. 1292-1298, 2010.

[24] W. Li, L. Zhao, and L. S. Kang, "Robust satisfactory faulttolerant design for uncertain nonlinear NCS with actuator fault," Journal of System Simulation, vol. 26, no. 11, pp. 26682681, 2014.

[25] Y. Li and W. Li, "Discrete event-triggered robust fault-tolerant control for nonlinear networked control systems with $\alpha$-safety degree and actuator saturation," Mathematical Problems in Engineering, vol. 2015, Article ID 590598, 17 pages, 2015.

[26] H.-C. Cao and W. Li, "Non-fragile robust fault-tolerant control for uncertain NCS with actuator saturation," Control and Decision, vol. 28, no. 12, pp. 1874-1883, 2013.

[27] F. S. Yang, H. G. Zhang, H. J. Gao, and S. Q. Wang, "Modeindependent fuzzy fault-tolerant variable sampling stabilization of nonlinear networked systems with both time-varying and random delays," Fuzzy Sets Systems, vol. 207, pp. 45-63, 2012.

[28] C. Peng and Q.-L. Han, "Delay-range-dependent robust stabilization for uncertain T-S fuzzy control systems with interval time-varying delays," Information Sciences, vol. 181, no. 19, pp. 4287-4299, 2011.

[29] K. Liu and E. Fridman, "Wirtinger's inequality and Lyapunovbased sampled-data stabilization," Automatica, vol. 48, no. 1, pp. 102-108, 2012.

[30] H.-C. Cao and W. Li, "Robust fault-tolerant control for nonlinear networked control system under discrete event-triggered communication scheme," Journal of Jilin University (Engineering and Technology Edition), vol. 45, no. 6, pp. 1895-1905, 2015.

[31] A. Eqtami, D. Dimarogonas, and K. Kyriakopoulos, "Eventtriggered control for discrete-time system," in Proceedings of the 2010 American Control Conference (ACC '10), pp. 4719-4724, Baltimore, Md, USA, 2010.

[32] W. Li and D. N. Jiang, "Robust $H_{\infty}$ fault-tolerant control for a nonlinear networked control system based on the TakagiSugeno fuzzy model," Control and Decision, vol. 25, no. 4, pp. 598-604, 2010. 


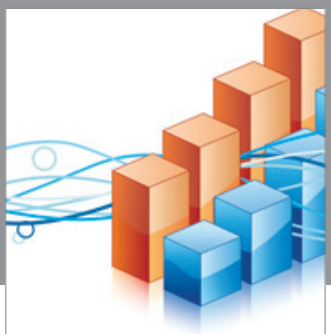

Advances in

Operations Research

vatem alat4

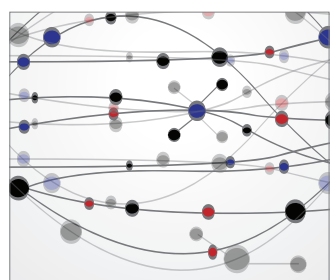

\section{The Scientific} World Journal
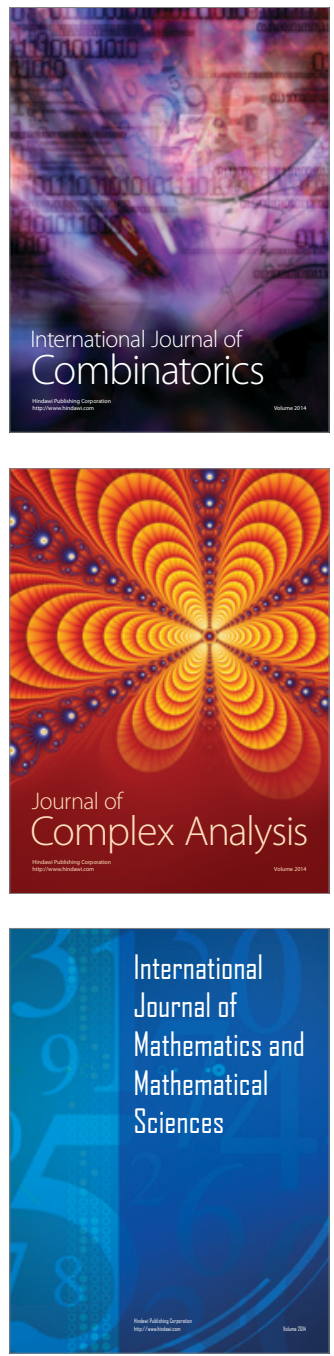
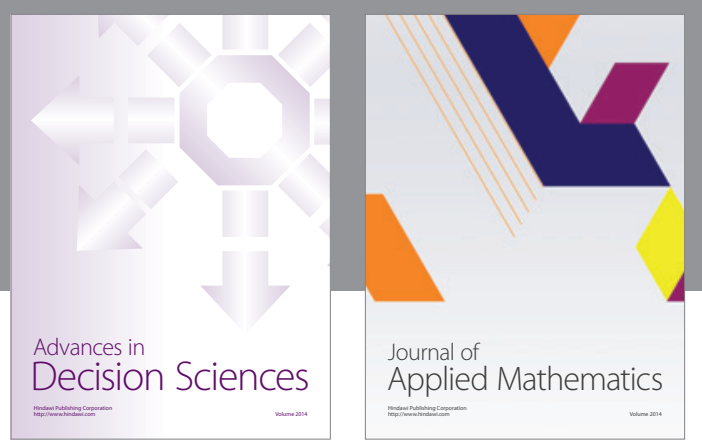

Algebra

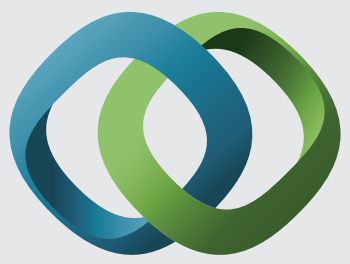

\section{Hindawi}

Submit your manuscripts at

http://www.hindawi.com
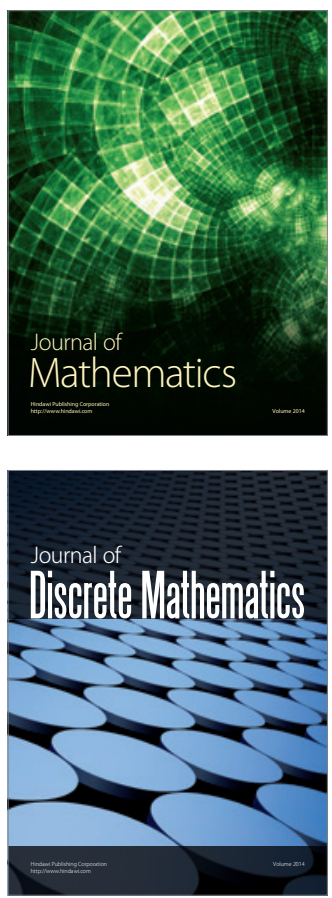

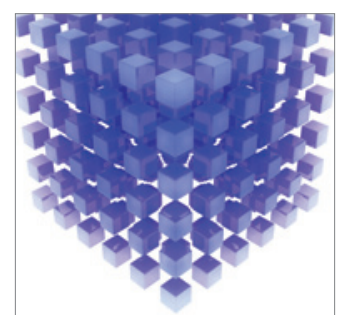

Mathematical Problems in Engineering
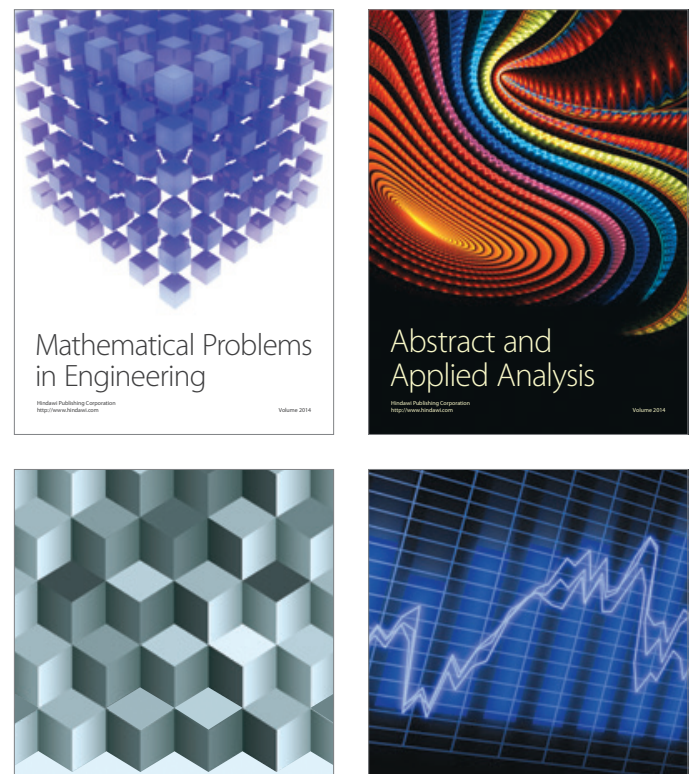

Journal of

Function Spaces

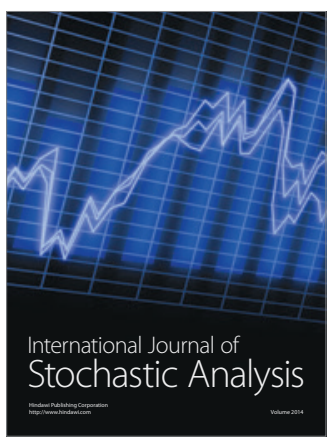

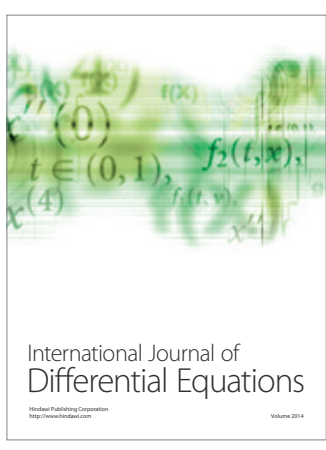
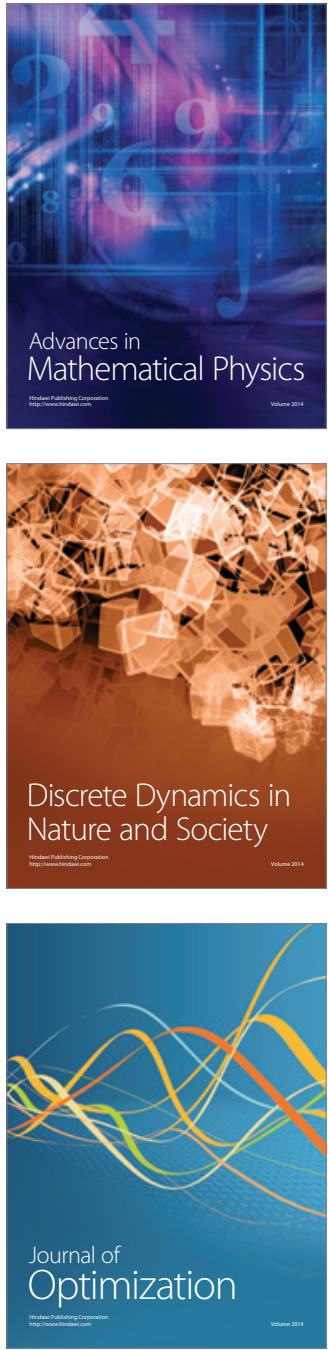